\title{
Projective structures, grafting and measured laminations
}

\author{
DAVID DUMAS \\ MichaEL WOLF
}

\begin{abstract}
We show that grafting any fixed hyperbolic surface defines a homeomorphism from the space of measured laminations to Teichmüller space, complementing a result of Scannell-Wolf on grafting by a fixed lamination. This result is used to study the relationship between the complex-analytic and geometric coordinate systems for the space of complex projective $\left(\mathbb{C} \mathbb{P}^{1}\right)$ structures on a surface.

We also study the rays in Teichmüller space associated to the grafting coordinates, obtaining estimates for extremal and hyperbolic length functions and their derivatives along these grafting rays.
\end{abstract}

30F60; 30F10, 30F40, 32G15, 57M50

\section{Introduction}

In this paper we compare two perspectives on the theory of complex projective structures on surfaces by studying the grafting map of a hyperbolic surface.

A complex projective (or $\mathbb{C P}^{1}$ ) structure on a compact surface $S$ is an atlas of charts with values in $\mathbb{C P} \mathbb{P}^{1}$ and Möbius transition functions. Let $\mathcal{P}(S)$ denote the space of (isotopy classes of) marked complex projective structures on $S$, and let $\mathcal{T}(S)$ be the Teichmüller space of (isotopy classes of) marked complex structures on $S$. Because Möbius maps are holomorphic, there is a forgetful projection $\pi: \mathcal{P}(S) \rightarrow \mathcal{T}(S)$.

An analytic tradition, having much in common with univalent function theory, parameterizes the fiber $\pi^{-1}(X)$ using the Schwarzian derivative, identifying $\mathcal{P}(S)$ with the total space of the bundle $\mathcal{Q}(S) \rightarrow \mathcal{T}(S)$ of holomorphic quadratic differentials.

A second, more synthetic geometric description of $\mathcal{P}(S)$ is due to Thurston, and proceeds through the operation of grafting - a construction which traces its roots back at least to Klein [25, Section 50], with a modern history developed by many authors (Maskit [27], Hejhal [19], Sullivan-Thurston [41], Goldman [18], Gallo-KapovichMarden [15], Tanigawa [42], McMullen [29] and Scannell-Wolf [36]). The simplest example of grafting may be described as follows. 
Start with a hyperbolic surface $X \in \mathcal{T}(S)$ and a simple closed geodesic $\gamma$ on $X$; then construct a new surface by removing $\gamma$ from $X$ and replacing it with the Euclidean cylinder $\gamma \times[0, t]$. The result is $\operatorname{Gr}_{t \gamma} X$, the grafting of $X$ by $t \gamma$, which is a surface with a ( $C^{1,1}$ Riemannian) metric composed of alternately flat or hyperbolic pieces. Furthermore, $\mathrm{Gr}_{t \gamma} X$ has a canonical projective structure that combines the Fuchsian uniformization of $X$ and the Euclidean structure of the cylinder $\gamma \times[0, t]$ (for details, see Scannell-Wolf [36, Section 1], Tanigawa [42, Section 2] and Kamishima-Tan [20]).

Thurston showed that grafting extends naturally from weighted simple closed geodesics to the space $\mathcal{M L}(S)$ of measured geodesic laminations, and thus defines a map

$$
\text { Gr: } \mathcal{M L}(S) \times \mathcal{T}(S) \rightarrow \mathcal{P}(S) .
$$

Moreover, this map is a homeomorphism; for a proof of this result, see [20].

A natural problem is to relate the analytic and geometric perspectives on the space of projective structures, for example by comparing the product structure of $\mathcal{M L}(S) \times$ $\mathcal{T}(S) \simeq \mathcal{P}(S)$ to the bundle structure induced by the projection $\pi: \mathcal{P}(S) \rightarrow \mathcal{T}(S)$.

\section{Results on grafting}

We compare these two perspectives on projective structures by studying the conformal grafting map $\mathrm{gr}=\pi \circ \mathrm{Gr}: \mathcal{M L}(S) \times \mathcal{T}(S) \rightarrow \mathcal{T}(S)$, ie $\mathrm{gr}_{\lambda} X$ is the conformal structure which underlies the projective structure $\mathrm{Gr}_{\lambda} X$. Fixing either of the two coordinates we have the $X$-grafting map gr. $X: \mathcal{M L}(S) \rightarrow \mathcal{T}(S)$ and the $\lambda$-grafting map $\operatorname{gr}_{\lambda}: \mathcal{T}(S) \rightarrow \mathcal{T}(S)$. These maps reflect how the base coordinate of the complexanalytic fibration $\pi: \mathcal{P}(S) \rightarrow \mathcal{T}(S)$ is related to the geometric product structure $\mathcal{M L}(S) \times \mathcal{T}(S) \simeq \mathcal{P}(S)$. Our main result is the following Theorem.

Theorem 1.1 For each $X \in \mathcal{T}(S)$, the $X$-grafting map gr. $X: \mathcal{M L}(S) \rightarrow \mathcal{T}(S)$ is a bitangentiable homeomorphism.

Momentarily deferring a brief discussion of the term bitangentiable homeomorphism, we note that this theorem is a natural complement to the result of Scannell-Wolf on the $\lambda$-grafting map.

Theorem 1.2 (Scannell-Wolf [36, Theorem A]) For each $\lambda \in \mathcal{M L}(S)$, the map $\mathrm{gr}_{\lambda}: \mathcal{T}(S) \rightarrow \mathcal{T}(S)$ is a real-analytic diffeomorphism.

The discrepancy between diffeomorphism and bitangentiable homeomorphism in Theorem 1.1 and Theorem 1.2 is related to the lack of a natural differentiable structure on 
$\mathcal{M L}(S)$. Bonahon showed that grafting is differentiable in the weak sense of being tangentiable; see Section 2 below or [7] for details.

Returning to the original problem of comparing different coordinate systems for $\mathcal{P}(S)$, Theorem 1.1 and Theorem 1.2 can be used to study the fiber $P(X)=\pi^{-1}(X)$ and its relation to the grafting coordinates. Let us denote the two factors of the map $\mathrm{Gr}^{-1}: \mathcal{P}(S) \rightarrow \mathcal{M L}(S) \times \mathcal{T}(S)$ by

$$
\operatorname{Gr}^{-1}(Z)=\left(p_{\mathcal{M L}}(Z), p_{\mathcal{T}}(Z)\right)
$$

Thus the maps $p_{\mathcal{M L}}: \mathcal{P}(S) \rightarrow \mathcal{M L}(S)$ and $p_{\mathcal{T}}: \mathcal{P}(S) \rightarrow \mathcal{T}(S)$ send a projective structure to one of its two grafting coordinates, and we think of them as projections. We prove the following Corollary.

Corollary 1.3 For each $X \in \mathcal{T}(S)$ the restriction $\left.p_{\mathcal{M L}}\right|_{P(X)}: P(X) \rightarrow \mathcal{M L}(S)$ is a bitangentiable homeomorphism, and $\left.p_{\mathcal{T}}\right|_{P(X)}: P(X) \rightarrow \mathcal{T}(S)$ is a $C^{1}$ diffeomorphism.

This corollary improves the existing regularity results for these projection maps, from which it was known that that $\left.p_{\mathcal{M L}}\right|_{P(X)}$ is a homeomorphism (a corollary of Theorem 1.2 , see Dumas [12, Section 4]) and that $\left.p_{\mathcal{T}}\right|_{P(X)}$ is a proper $C^{1}$ map of degree 1 (see Bonahon [7, Theorem 3] and Dumas [11, Lemma 7.6, Theorem 1.1]). The relationship between the maps $p_{\mathcal{T}}, p_{\mathcal{M L}}$, and $\pi$ is represented schematically in Figure 1.

One can also apply Theorem 1.1 and Corollary 1.3 to study the pruning map (the inverse of grafting) and the parameterization of quasi-Fuchsian manifolds by their convex hull geometry. We explore these directions in Section 5.

\section{Methods}

From a general perspective, the proof of the main theorem relies on relating two established techniques in hyperbolic geometry. The first is the analytic study of the prescribed curvature (Liouville) equation (see eg Wolpert [51], Zograf-Takhtadzhyan [52] and Tromba [46]), and the second is the complex duality between bending and twisting (see eg Wolpert [48; 49; 50], Platis [34] and Series [37; 38]) and its generalization to complex earthquakes as a duality between grafting and shearing (see Bonahon [7], McMullen [29] and Epstein-Marden-Markovic [14]).

The first strand occurs in the proof in Scannell-Wolf [36] of Theorem 1.2, where standard geometric analytic techniques were applied to the curvature equation to understand how the $\lambda$-grafting map changed under small perturbations of the Riemann surface. Such techniques might be applicable to the analogous problem (of the main Theorem 


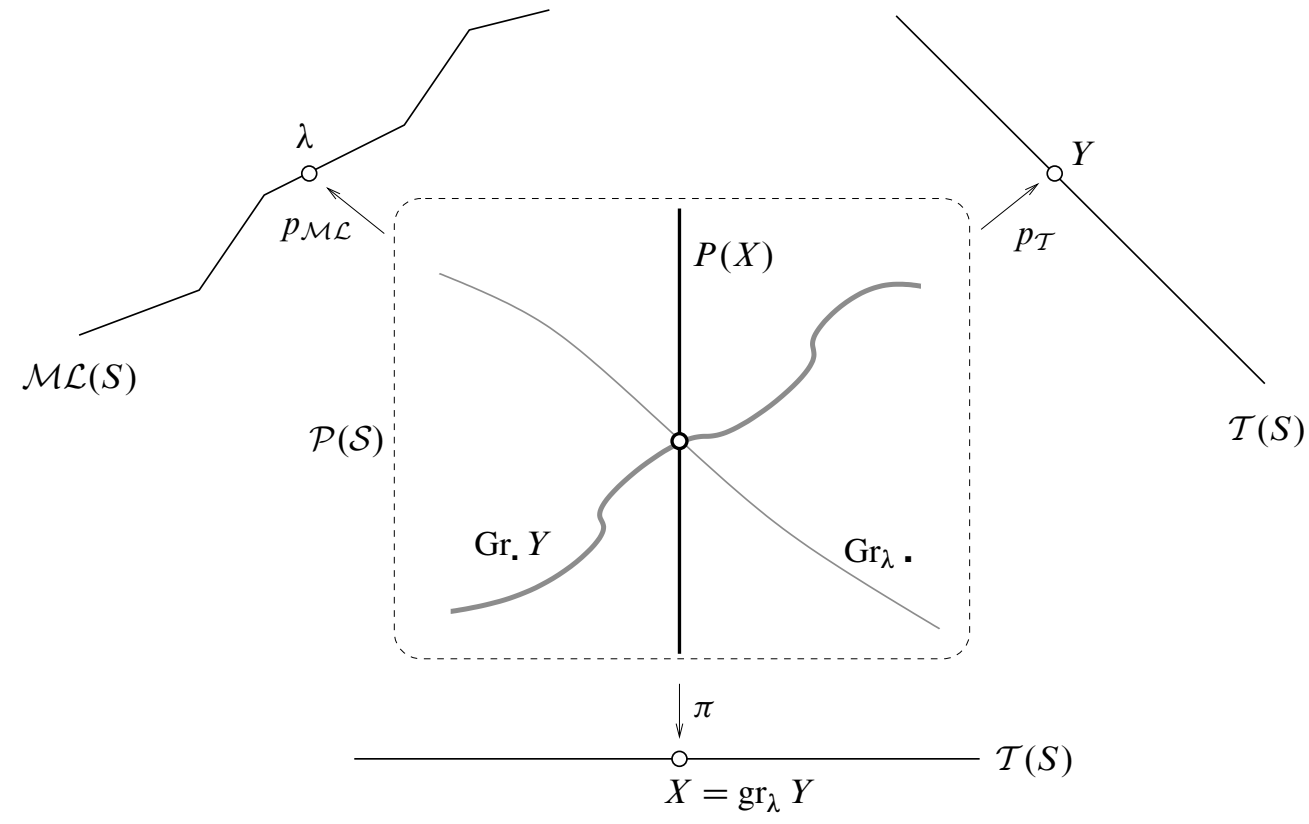

Figure 1: The bundle $\pi: \mathcal{P}(S) \rightarrow \mathcal{T}(S)$ of $\mathbb{C P}^{1}$ structures over Teichmüller space and the product structure $\operatorname{Gr}: \mathcal{M L}(S) \times \mathcal{T}(S) \simeq \mathcal{P}(S)$ induced by grafting.

1.1) of understanding how the $X$-grafting map varies under small perturbations of the measured lamination, but it would necessarily be more involved, due to the local structure of the space $\mathcal{M L}(S)$ being more complicated than that of the space $\mathcal{T}(S)$.

Fortunately, most of the required details for this study of $\mathcal{M L}(S)$ are already in the literature: here we make heavy use of Bonahon's work (following Thurston [43]) on the deformation theory of $\mathcal{M L}(S)$ (see Bonahon $[4 ; 5 ; 6 ; 7]$ ). In particular, the crux of our proof relies on Bonahon's observation that there is a sense in which infinitesimal grafting is complex linear. This complex linearity, in keeping with the second tradition discussed above, implies that a study of the effect on grafting of infinitesimally changing the measured lamination is, by duality, a study of the effect on grafting of infinitesimally shearing the hyperbolic surface. Thus we may apply the analysis in the proof of Theorem 1.2 to the problem of the main Theorem 1.1.

\section{Grafting rays}

In a final section, we study the coordinate system on Teichmüller space induced by the $X$-grafting homeomorphism $\mathcal{M L}(S) \rightarrow \mathcal{T}(S)$, and analyze the behavior of 
extremal and hyperbolic length functions on grafting rays-paths in $\mathcal{T}(S)$ of the form $t \mapsto \operatorname{gr}_{t \lambda} X$. We prove the following theorems.

Theorem 1.4 For each $X \in \mathcal{T}(S)$ and $\lambda \in \mathcal{M L}(S)$, the extremal length of $\lambda$ on $\operatorname{gr}_{t \lambda} X$ is monotone decreasing for all $t \gg 0$ and is asymptotic to $\frac{\ell(\lambda, X)}{t}$, where $\ell(\lambda, X)$ is the hyperbolic length of $\lambda$ on $X$.

Theorem 1.5 For each $X \in \mathcal{T}(S)$ and any simple closed hyperbolic geodesic $\gamma \in$ $\mathcal{M L}(S)$, the hyperbolic length of $\gamma$ on $\operatorname{gr}_{t \gamma} X$ is monotone decreasing for all $t \gg 0$ and is asymptotic to $\pi \frac{\ell(\lambda, X)}{t}$.

The monotonicity and asymptotic behavior described in Theorem 1.4 and Theorem 1.5 are combined with explicit estimates on the derivatives of length functions in Theorem 6.2 and Theorem 6.6 below.

\section{Organization of the paper}

Section 2 presents the infinitesimal version of the main theorem (Theorem 2.6), after introducing the necessary background on measured laminations and grafting. The reduction to an infinitesimal statement is modeled on the argument of Scannell-Wolf in [36], and uses Tanigawa's properness theorem for grafting (Theorem 2.1).

Section 3 describes shearing deformations of hyperbolic surfaces, closely following the work of Bonahon on shearing coordinates for Teichmüller space. Portions of this section are more expository than is strictly necessary for the proofs of the of the results, but we feel they help make the arguments easier to understand. The discussion culminates with the crucial complex-linearity result of Bonahon (Theorem 3.6) that is used in the proof of Theorem 2.6.

Section 4 is devoted to the proofs of Theorem 2.6 and Theorem 1.1, which follow using the theory developed in Section 2 and Section 3.

Section 5 collects some applications of the main theorem, including the proof of Corollary 1.3 and a rigidity result for quasi-Fuchsian manifolds.

Section 6 discusses the grafting coordinates for Teichmüller space and the asymptotic behavior of extremal and hyperbolic length functions on grafting rays; Theorem 1.4 and Theorem 1.5 and associated derivative estimates are proved here. 


\section{Acknowledgements}

The authors thank Francis Bonahon, Howard Masur, Yair Minsky, and Robert Penner for stimulating discussions related to this work. They also thank the referee for several suggestions that improved the paper.

The first author was partially supported by an NSF postdoctoral research fellowship. The second author was partially supported by NSF grants DMS-0139877 and DMS0505603.

\section{Grafting and infinitesimal grafting}

We begin with some background on measured laminations, grafting, and tangentiability, which are needed to formulate the main technical result (Theorem 2.6).

\section{Laminations}

As in the introduction, $S$ denotes a compact smooth surface of genus $g>1$ and $\mathcal{T}(S)$ is the Teichmüller space of marked hyperbolic (or conformal) structures on $S$. We often use $X \in \mathcal{T}(S)$ to represent a particular hyperbolic surface in a given marked equivalence class.

Let $\mathcal{S}$ denote the set of free homotopy classes of simple closed curves on $S$; we implicitly identify $\gamma \in \mathcal{S}$ with its geodesic representative on a hyperbolic surface $X \in \mathcal{T}(S)$.

A geodesic lamination $\Lambda$ on a hyperbolic surface $X$ is a foliation of a closed subset of $X$ by complete, simple geodesics. Examples of geodesic laminations include simple closed hyperbolic geodesics $\gamma \in \mathcal{S}$ and disjoint unions thereof.

The notion of a geodesic lamination is actually independent of the particular choice of $X$, in that a geodesic lamination on $X$ determines a geodesic lamination for any other hyperbolic structure $Y \in \mathcal{T}(S)$ in a canonical way (see for example Bonahon [5, Section 1]). Thus we speak of a geodesic lamination on $S$, suppressing the choice of a particular metric. Let $\mathcal{G} \mathcal{L}(S)$ denote the set of all geodesic laminations on $S$ with the topology of Hausdorff convergence of closed sets.

A geodesic lamination $\Lambda \in \mathcal{G L}(S)$ is maximal if it is not properly contained in another geodesic lamination, in which case the complement of $\Lambda$ in $S$ is a union of ideal triangles. Every geodesic lamination is contained in a maximal one, though not necessarily uniquely. 


\section{Measured laminations}

A transverse measure $\mu$ on a geodesic lamination $\Lambda$ is an assignment of a positive Borel measure to each compact transversal to $\Lambda$ in a manner compatible with splitting and isotopy of transversals. Such a measure $\mu$ has full support if there is no proper sublamination $\Lambda^{\prime} \subset \Lambda$ such that $\mu$ assigns the zero measure to transversals disjoint from $\Lambda^{\prime}$.

Let $\mathcal{M L}(S)$ denote the space of measured geodesic laminations on $S$, ie pairs $\lambda=$ $(\Lambda, \mu)$ where $\Lambda \in \mathcal{G} \mathcal{L}(S)$ and $\mu$ is a transverse measure on $\Lambda$ of full support. We denote by $\lambda(\tau)$ the total measure assigned to a transversal $\tau$ by $\lambda \in \mathcal{M L}(S)$.

The topology on $\mathcal{M L}(S)$ is that of weak-* convergence of measures on compact transversals. The underlying geodesic lamination of $\lambda \in \mathcal{M L}(S)$ is the support of $\lambda$, written $\operatorname{supp}(\lambda) \in \mathcal{G L}(S)$. The space $\mathcal{M L}(S)$ has an action of $\mathbb{R}^{+}$by multiplication of transverse measures; the empty lamination $0 \in \mathcal{M L}(S)$ is the unique fixed point of this action.

For any simple closed geodesic $\gamma \in \mathcal{S}$, there is a measured geodesic lamination (also $\gamma$ ) that assigns to a transversal $\tau$ the counting measure on $\tau \cap \gamma$. The rays $\left\{t \gamma \mid t \in \mathbb{R}^{+}, \gamma \in \mathcal{S}\right\}$ determined by simple closed curves are dense in $\mathcal{M L}(S)$.

The space $\mathcal{M L}(S)$ is a contractible topological manifold homeomorphic to $\mathbb{R}^{6 g-6}$, but it does not have a natural smooth structure. Its natural structure is that of a piecewise linear (PL) manifold, with charts corresponding to train tracks.

Detailed discussion of the space $\mathcal{M L}(S)$ can be found in Thurston [44], EpsteinMarden [13], Penner-Harer [33] and Otal [31].

\section{Grafting}

As mentioned in the introduction, Thurston showed that grafting along simple closed curves has a natural extension to measured laminations, giving a projective grafting homeomorphism Gr: $\mathcal{M L}(S) \times \mathcal{T}(S) \rightarrow \mathcal{P}(S)$ and a conformal grafting map gr: $\mathcal{M L}(S) \times \mathcal{T}(S) \rightarrow \mathcal{T}(S)$. Tanigawa showed that the latter is a proper map when either one of the two parameters is fixed.

Theorem 2.1 (Tanigawa [42]) The $\lambda$-grafting map $\operatorname{gr}_{\lambda}: \mathcal{T}(S) \rightarrow \mathcal{T}(S)$ is proper for any $\lambda \in \mathcal{M L}(S)$. For any $X \in \mathcal{T}(S)$, the $X$-grafting gr. $X: \mathcal{M L}(S) \rightarrow \mathcal{T}(S)$ is proper.

The properness of the restricted grafting maps is used in the proofs of Theorem 1.1 (in Section 4) and Theorem 1.2 (in Scannell-Wolf [36]) to reduce a global statement to a 
local one, which is then attacked using infinitesimal methods. In the case of $\lambda$-grafting, the infinitesimal analysis is possible because $\mathrm{gr}_{\lambda}: \mathcal{T}(S) \rightarrow \mathcal{T}(S)$ is differentiable, and even real-analytic (see McMullen [29, Corollary 2.11]). A related real-analyticity property along rays in $\mathcal{M L}(S)$ is discussed in Section 6.

The main step in [36] is to show that the differential map $d \mathrm{gr}_{\lambda}: T_{X} \mathcal{T}(S) \rightarrow T_{\mathrm{gr}_{\lambda} X} \mathcal{T}(S)$ is an isomorphism. Once that is established, Theorem 1.2 follows easily, since $\operatorname{gr}_{\lambda}$ is then a proper local diffeomorphism of $\mathcal{T}(S)$, hence a covering map of the simply connected space $\mathcal{T}(S)$.

We will follow an analogous outline in the proof of Theorem 1.1, but the infinitesimal analysis is complicated by lack of smooth structure on $\mathcal{M L}(S)$, so the derivative of $X$-grafting does not exist in the classical sense. Instead we must use a weaker notion of differentiability based on one-sided derivatives, which we now discuss.

\section{Tangentiability}

A tangentiable map ${ }^{1} f: U \rightarrow V$ between open sets in $\mathbb{R}^{n}$ is a map with "one-sided" directional derivatives everywhere; in other words, for each $x \in U$ and $v \in \mathbb{R}^{n}$, the limit

$$
\left.\frac{d}{d t}\right|_{t=0^{+}} f(x+t v)=\lim _{t \rightarrow 0^{+}} \frac{f(x+t v)-f(x)}{t}
$$

exists, and the convergence is locally uniform in $v$ (for equivalent conditions, see [7, Section 2]). This convergence allows us to define $T_{x} f: \mathbb{R}^{n} \rightarrow \mathbb{R}^{n}$, the tangent map of fat $x$, by

$$
T_{x} f(v)=\left.\frac{d}{d t}\right|_{t=0^{+}} f(x+t v) .
$$

Of course if $f$ is differentiable, then $T_{x} f$ is just the derivative of $f$ at $x$, a linear map. When $f$ is only tangentiable, the map $T_{x} f$ is continuous and homogeneous in the sense that $T_{x} f(\lambda v)=\lambda T_{x} f(v)$ for $\lambda \in \mathbb{R}^{+}[7$, Section 1].

A tangentiable manifold is one whose transition functions are tangentiable maps; examples include smooth manifolds and PL manifolds. Thus $\mathcal{M L}(S)$ has a natural tangentiable structure. The tangent space $T_{x} M$ at a point $x$ of a tangentiable manifold is not naturally a vector space, but has the structure of a cone. The notion of tangentiable map extends naturally to tangentiable manifolds.

We will say that a homeomorphism between two tangentiable manifolds is a bitangentiable homeomorphism if it and its inverse are tangentiable, and if the tangent maps are everywhere homeomorphisms. A convenient criterion for this is provided by the following Lemma.

\footnotetext{
${ }^{1}$ Some authors say instead that the map is one-sided Gateaux differentiable.
} 
Lemma 2.2 (Bonahon [7, Lemma 4]) Let $f: M \rightarrow N$ be a homeomorphism between tangentiable manifolds. If $f$ is tangentiable, and all of its tangent maps are injective, then $f$ is a bitangentiable homeomorphism.

Bonahon showed that grafting is compatible with that tangentiable structure of $\mathcal{M L}(S)$ in the sense of the following Theorem.

Theorem 2.3 (Bonahon [7, Theorem 3]) The grafting map Gr: $\mathcal{M L}(S) \times \mathcal{T}(S) \rightarrow$ $\mathcal{P}(S)$ is a bitangentiable homeomorphism. In particular, the conformal grafting map gr: $\mathcal{M L}(S) \times \mathcal{T}(S) \rightarrow \mathcal{T}(S)$ is tangentiable, and for each $X \in \mathcal{T}(S)$, the $X$-grafting map gr. $X: \mathcal{M L}(S) \rightarrow \mathcal{T}(S)$ is tangentiable.

In [7], Bonahon actually computes the tangent map of grafting to show that grafting is tangentiable. After developing the shearing coordinates in Section 3, Bonahon's description of the tangent map (from which Theorem 2.3 is derived) is given in Theorem 3.6.

\section{Differentiability}

A curious feature of the tangentiability of grafting with respect to $\mathcal{M L}(S)$ is that some fragments of classical differentiability remain. For example, the inverse of the projective grafting map $\mathrm{Gr}^{-1}: \mathcal{P}(S) \rightarrow \mathcal{M L}(S) \times \mathcal{T}(S)$ factors into the two projections $p_{\mathcal{M L}}$ and $p_{\mathcal{T}}$ (as described in the introduction). By Theorem 2.3, these are also tangentiable maps, but since both the domain and range of $p_{\mathcal{T}}$ are smooth manifolds, it makes sense to ask if this map is differentiable in the usual sense. Extending Theorem 2.3, Bonahon proves the following Theorem.

Theorem 2.4 (Bonahon [7, Theorem 3]) The map $p_{\mathcal{T}}: \mathcal{P}(S) \rightarrow \mathcal{T}(S)$ is $C^{1}$.

In the same article, Bonahon shows that $p_{\mathcal{T}}$ fails to be $C^{2}$ for a certain family of punctured torus groups, which suggests that $p_{\mathcal{T}}$ may fail to be $C^{2}$ for all Teichmüller spaces $\mathcal{T}(S)$.

Finally we observe that $p_{\mathcal{T}}\left(\operatorname{Gr}_{\lambda} X\right)=X$, so for each $\lambda \in \mathcal{M L}(S)$ we have $p_{\mathcal{T}}$ 。 $\mathrm{Gr}_{\lambda}=\mathrm{Id}$, that is, the map $\mathrm{Gr}_{\lambda}: \mathcal{T}(S) \rightarrow \mathcal{P}(S)$ is a smooth section of $p_{\mathcal{T}}$. Since Gr: $\mathcal{M L}(S) \times \mathcal{T}(S) \rightarrow \mathcal{P}(S)$ is a homeomorphism, these sections fill up $\mathcal{P}(S)$, and we obtain the following Corollary.

Corollary 2.5 The map $p_{\mathcal{T}}$ is a $C^{1}$ submersion. 


\section{Infinitesimal $X$-grafting}

Using the tangentiability of grafting, we can formulate the infinitesimal statement that will be our main tool in the proof of Theorem 1.1.

Theorem 2.6 The tangent map $T_{\lambda}$ gr. $X$ of the $X$-grafting map has no kernel. That is, if $\lambda_{t}$ is a tangentiable family of measured laminations and $\left.\frac{d}{d t}\right|_{t=0^{+}} \operatorname{gr}_{\lambda_{t}} X=0$, then $\left.\frac{d}{d t}\right|_{t=0^{+}} \lambda_{t}=0$.

In the next two sections, we develop machinery to prove this result about the derivative of grafting, then strengthen it to a local injectivity result in order to prove Theorem 1.1. Complications arise in both steps because the maps under consideration are tangentiable rather than smooth.

\section{Shearing}

In this section we describe the machinery of shearing cocycles for geodesic laminations on a hyperbolic surface, borrowing heavily from the papers of Bonahon [6; 4]. Some examples and discussion are included here to clarify the technicalities that our later arguments will require.

\section{Cocycles}

Let $G$ be an abelian group and let $\Lambda \in \mathcal{G L}(S)$. A $G$-valued cocycle on $\Lambda$ is a map $\alpha$ that assigns to each transversal $\tau$ to $\Lambda$ an element $\alpha(\tau) \in G$ in a manner compatible with splitting and transversality-preserving isotopy. The $G$-module of all $G$-valued cocycles on $\Lambda$ is denoted $\mathcal{H}(\Lambda, G)$.

Of particular interest for our purposes is the vector space of cocycles for maximal laminations with values in $\mathbb{R}$. While it is perhaps not clear from the definition, this vector space is finite dimensional, and the dimension is the same for all maximal laminations.

Theorem 3.1 (Bonahon [4, Proposition 1]) Let $\Lambda \in \mathcal{G} \mathcal{L}(S)$ be a maximal lamination. Then $\mathcal{H}(\Lambda, \mathbb{R}) \simeq \mathbb{R}^{6 g-6}$.

The vector space $\mathcal{H}(\Lambda, \mathbb{R})$ carries a natural alternating bilinear form $\omega: \mathcal{H}(\Lambda, \mathbb{R}) \times$ $\mathcal{H}(\Lambda, \mathbb{R}) \rightarrow \mathbb{R}$, the Thurston symplectic form, which comes from the cup product on $H^{1}(S, \mathbb{R})$ (see Penner-Harer [33], Bonahon [4, Section 3] and Sözen-Bonahon [40]). 
When $\Lambda$ is maximal, the form $\omega$ is nondegenerate, making $\mathcal{H}(\Lambda, \mathbb{R})$ a symplectic vector space. While we do not use this symplectic structure directly in the proof of the main theorem, it is relevant to some of the constructions and examples in the sequel.

If $\lambda \in \mathcal{M L}(S)$ is a measured lamination with $\operatorname{supp}(\lambda) \subset \Lambda \in \mathcal{G L}(S)$, then the total measure $\tau \mapsto \lambda(\tau)$ defines a real-valued cocycle on $\Lambda$, which we also denote by $\lambda \in \mathcal{H}(\Lambda, \mathbb{R})$. Cocycles arising from measures in this way take only nonnegative values on transversals; Bonahon showed that the converse is also true.

Theorem 3.2 (Bonahon [6, Proposition 18]) A transverse cocycle $\alpha \in \mathcal{H}(\Lambda, \mathbb{R})$ arises from a transverse measure for $\Lambda$ if and only if $\alpha(\tau) \geq 0$ for every transversal $\tau$.

We therefore define $\mathcal{M}(\Lambda) \subset \mathcal{H}(\Lambda, \mathbb{R})$, the cone of transverse measures for $\Lambda$, to be the set of cocycles $\alpha$ satisfying $\alpha(\tau) \geq 0$ for all transversals $\tau$. The set $\mathcal{M}(\Lambda)$ is a convex cone in the vector space $\mathcal{H}(\Lambda, \mathbb{R})$.

While positive real-valued cocycles on $\Lambda$ correspond to transverse measures, there is an essential difference between a real-valued cocycle $\alpha \in \mathcal{H}(\Lambda, \mathbb{R})$ (whose value on a transversal is a real number) and a signed transverse measure on $\Lambda$ which assigns a countably additive signed measure to each transversal $\tau$ (see Examples 3.4-3.5 below).

An analogous situation is the set function $[a, b] \mapsto(f(b)-f(a))$, where $f$ is a real-valued function; this is a finitely additive function on intervals, but it only arises from a signed Borel measure if $f$ has bounded variation (which is automatic if $f$ is monotone). The connection between this example and real-valued cocycles for a lamination can be seen through the "distribution function" $f(x)=\alpha\left(\tau_{x}\right)$ where $\alpha \in \mathcal{H}(\Lambda, \mathbb{R}), \tau:[0,1] \rightarrow S$ is a transversal, and $\tau_{x}=\left.\tau\right|_{[0, x]}$. This function is defined for a.e. $x \in[0,1]$ and monotonicity (for every $\tau$ ) is equivalent to $\alpha$ being nonnegative.

The difference between measures and cocycles is also apparent from the dimension of the span of $\mathcal{M}(\Lambda)$, which is often 1 (by the solution to the Keane conjecture, see Masur [28] Veech [47], Rees [35] and Kerckhoff [24]) and is never more than (3g-3) (because for maximal $\Lambda$, the space $\mathcal{H}(\Lambda, \mathbb{R}) \simeq \mathbb{R}^{6 g-6}$ is a symplectic vector space in which $\operatorname{span}(\mathcal{M}(\Lambda))$ is isotropic, see Papadopoulos [32], Levitt [26] and Katok [21]). It follows that $\mathcal{M}(\Lambda)$ has positive codimension when, for example, $\Lambda$ is maximal.

\section{Shearing coordinates}

Let $\Lambda \in \mathcal{G L}(S)$ be a maximal lamination, realized as a partial foliation of $X \in \mathcal{T}(S)$ by hyperbolic geodesics. Its lift $\widetilde{\Lambda}$ to the universal cover $\widetilde{X} \simeq \mathbb{H}^{2}$ determines a (not necessarily locally finite) tiling of $\mathbb{H}^{2}$ by ideal triangles. 
A transversal $\tau:[a, b] \rightarrow \mathbb{H}^{2}$ to $\widetilde{\Lambda}$ determines a pair of ideal triangles $T_{a}$ and $T_{b}$ which are the complementary regions of $\widetilde{\Lambda}$ containing $\tau(a)$ and $\tau(b)$. In [4], Bonahon constructs a shearing cocycle $\sigma(X)=\sigma^{\Lambda}(X) \in \mathcal{H}(\Lambda, \mathbb{R})$ from these data with the property that $\sigma(X)(\tau)$ measures the "relative shear" of the triangles $T_{a}$ and $T_{b}$ in $\mathbb{H}^{2}$. For example, the relative shear of two ideal triangles that share an edge is the signed distance between the feet of the altitudes based on the common side.

Remarkably, $\sigma(X)$ determines the metric $X$, and the set of such cocycles admits an explicit description. Let $\mathcal{C}(\Lambda) \subset \mathcal{H}(\Lambda, \mathbb{R})$ denote the set of $\mathbb{R}$-valued cocycles that arise as shearing cocycles of hyperbolic metrics, and recall that $\omega: \mathcal{H}(\Lambda, \mathbb{R}) \times \mathcal{H}(\Lambda, \mathbb{R}) \rightarrow \mathbb{R}$ is the Thurston symplectic form.

Theorem 3.3 (Bonahon [4, Theorems A and B]) A cocycle $\alpha \in \mathcal{H}(\Lambda, \mathbb{R})$ is the shearing cocycle of a hyperbolic metric if and only if only if $\omega(\alpha, \mu)>0$ for all $\mu \in \mathcal{M}(\Lambda)$, and $\mathcal{C}(\Lambda)$ is an open convex cone with finitely many faces. Furthermore $\sigma: \mathcal{T}(S) \rightarrow \mathcal{C}(\Lambda)$ is a real-analytic diffeomorphism.

The condition $\omega(\alpha, \mu)>0$ in Theorem 3.3 is necessary because for each $\mu \in \mathcal{M}(\Lambda)$, the Thurston pairing $\omega(\sigma(X), \mu)$ is the hyperbolic length of $\mu$ on $X$ [4, Theorem 9].

While the convex cone $\mathcal{C}(\Lambda)$ has finitely many faces and is a union of open rays in $\mathcal{H}(\Lambda, \mathbb{R})$, the zero cocycle $0 \in \mathcal{H}(\Lambda, \mathbb{R})$ is not an extreme point of $\mathcal{C}(\Lambda)$. In fact, the vector space of signed transverse measures (ie $\operatorname{span} \mathcal{M}(\Lambda)$ ) is $\omega$-isotropic (see Papadopoulos [32]), and therefore

$$
\operatorname{span} \mathcal{M}(\Lambda) \subset \partial \mathcal{C}(\Lambda)
$$

A schematic representation $\mathcal{C}(\Lambda)$ appears in Figure 2, where $\partial \mathcal{C}(\Lambda)$ contains a onedimensional subspace of $\mathcal{H}(\Lambda, \mathbb{R})$.

Remark Thurston parameterizes $\mathcal{T}(S)$ by a convex cone of shear coordinates in [43, Section 9]. The setting is in terms of duals to weights on train tracks, but the results are equivalent to those in Theorem 3.3.

\section{Shearing maps}

We now use the shearing embedding $\sigma: \mathcal{T}(S) \rightarrow \mathcal{H}(\Lambda, \mathbb{R})$ to turn translation in the vector space $\mathcal{H}(\Lambda, \mathbb{R})$ into a (locally-defined) map of Teichmüller space.

Let $X \in \mathcal{T}(S)$ and $\alpha \in \mathcal{H}(\Lambda, \mathbb{R})$. If the sum $\sigma(X)+\alpha$ is the shearing cocycle of a hyperbolic surface, we call this hyperbolic surface $\operatorname{sh}_{\alpha} X$, the shearing of $X$ by $\alpha$. Thus $\operatorname{sh}_{\alpha} X$ is defined by the condition

$$
\sigma\left(\operatorname{sh}_{\alpha} X\right)=\sigma(X)+\alpha .
$$




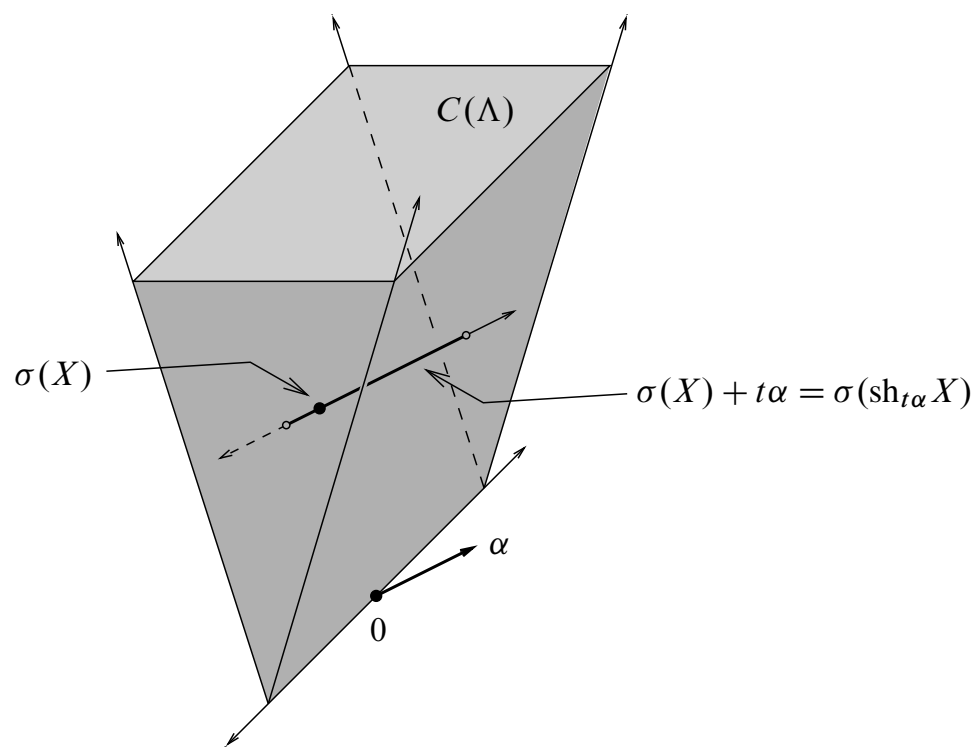

Figure 2: The shearing map is a translation in the shearing embedding of $\mathcal{T}(S)$ in $\mathcal{H}(\Lambda, \mathbb{R})$.

Since $\sigma(\mathcal{T}(S))=\mathcal{C}(\Lambda)$ is open, there is a neighborhood $U \subset \mathcal{H}(\Lambda, \mathbb{R}) \times \mathcal{T}(S)$ of $\{0\} \times \mathcal{T}(S)$ in which the shearing map sh: $U \rightarrow \mathcal{T}(S)$ is well-defined.

In particular, for any fixed $X \in \mathcal{T}(S)$ and $\alpha \in \mathcal{H}(\Lambda, \mathbb{R})$ there is some $\epsilon>0$ such that $\operatorname{sh}_{t \alpha} X$ is defined for all $|t|<\epsilon$ (see Figure 2), and

$$
\frac{d}{d t} \sigma\left(\operatorname{sh}_{t \alpha} X\right)=\alpha .
$$

We consider a few examples of shearing maps to highlight the role of $\mathcal{C}(\Lambda)$ and the fact that $\operatorname{sh}_{\alpha} X$ is not defined for all pairs $(\alpha, X)$. First, if $\Lambda$ contains a simple closed geodesic $\gamma$, then for each $t \in \mathbb{R}$ the cocycle $t \gamma$ is a signed transverse measure. Furthermore, for all $X \in \mathcal{T}(S)$, we have $(\sigma(X)+t \gamma) \in \mathcal{C}(\Lambda)$, since $t \gamma \subset \mathcal{M}(\Lambda)$ and the span of $\mathcal{M}(\Lambda)$ is $\omega$-isotropic; thus $\operatorname{sh}_{t \gamma} X$ is defined for all $t \in \mathbb{R}$. Concretely, the hyperbolic surface $\operatorname{sh}_{t \gamma} X$ is obtained from $X$ by cutting along the geodesic $\gamma$ and then gluing the two boundary components with a twist (by signed distance $t$ ).

This twisting example has a natural generalization: given a cocycle $\lambda \in \mathcal{H}(\Lambda, \mathbb{R})$ representing a transverse measure for $\Lambda$, the shearing $\operatorname{sh}_{t \lambda}(X)$ is again defined for all $t \in \mathbb{R}$ and the resulting map $\operatorname{sh}_{t \lambda}: \mathcal{T}(S) \rightarrow \mathcal{T}(S)$ is called an earthquake. For further discussion of earthquakes, see Thurston [45], Kerckhoff [23], Epstein-Marden [13], Bonahon [3] and McMullen [29]. 
As a final example, consider shearing a surface $X$ using a cocycle $\alpha \in \mathcal{H}(\Lambda, \mathbb{R})$ that is itself the shearing cocycle of a hyperbolic surface, ie $\alpha \in \mathcal{C}(\Lambda)$. For any transverse measure $\lambda \in \mathcal{M}(\Lambda)$, the hyperbolic length of $\lambda$ on $\operatorname{sh}_{t \alpha} X$ can be computed using the Thurston intersection form $\omega$ (cf [4, Theorem 9]), and we have

$$
\ell\left(\lambda, \operatorname{sh}_{t \alpha} X\right)=\omega(\sigma(X)+t \alpha, \lambda)=\ell(\lambda, X)+t \omega(\alpha, \lambda)=A+B t
$$

where $A, B>0$. Since the length of a measured lamination is positive, it follows that the set of $t$ for which $\sigma(X)+t \alpha$ is the shearing cocycle of a hyperbolic metric (and hence those for which $\operatorname{sh}_{t \alpha} X$ exists) is a subset of $\{t>-(A / B)\}$.

\section{Tangent cocycles}

Let $\lambda_{t} \in \mathcal{M L}(S), t \in[0, \epsilon)$ be a tangentiable ray of measured laminations. We will represent the tangent vector $\left.\frac{d}{d t}\right|_{t=0^{+}} \lambda_{t}$ by a transverse cocycle to a certain geodesic lamination (as in [5]). We first describe the underlying geodesic lamination.

The essential support of $\lambda_{t}$ at $t=0^{+}$is a geodesic lamination $\Lambda \in \mathcal{G} \mathcal{L}(S)$ that reflects how the support of $\lambda_{t}$ is changing for small positive values of $t$. For a $P L$ family of measured laminations $\lambda_{t}$, the essential support is the Hausdorff $\operatorname{limit}_{t \rightarrow 0^{+}} \operatorname{supp}\left(\lambda_{t}\right)$ of the supporting geodesic laminations [5, Proposition 4]. For the general case, we only sketch the construction, and refer the reader to [5, Section 2] for details.

First lift $\lambda_{t}$ to a family $\widetilde{\lambda}_{t}$ of measured geodesic laminations in $\mathbb{H}^{2}$. Define a set $\widetilde{\Lambda}$ of geodesics in $\mathbb{H}^{2}$ as follows: a geodesic $\gamma$ belongs to $\widetilde{\Lambda}$ if and only if for every smooth transversal $\tau:[-\epsilon, \epsilon] \rightarrow \mathbb{H}^{2}$ with $\tau(0) \in \gamma$, the total transverse measure of $\tau$ with respect to $\lambda_{t}$ is at least $C t$ for some $C>0$ (depending on $\tau$ and $\gamma$ ) and all $t$ sufficiently small. Then $\widetilde{\Lambda}$ is the lift of a geodesic lamination $\Lambda \in \mathcal{G} \mathcal{L}(S)$, the essential support of $\lambda_{t}$.

The tangent vector $\left.\frac{d}{d t}\right|_{t=0^{+}} \lambda_{t}$ defines a real-valued transverse cocycle $\dot{\lambda} \in \mathcal{H}(\Lambda, \mathbb{R})$ on the essential support as follows:

$$
\dot{\lambda}(\tau)=\lim _{t \rightarrow 0^{+}} \frac{1}{t}\left(\lambda_{t}(\tau)-\lambda_{0}(\tau)\right) .
$$

This is the tangent cocycle of $\lambda_{t}$ at $t=0^{+}$. Clearly the same formula defines a cocycle for a lamination containing the essential support of $\lambda_{t}$, so as a convenience we may assume $\Lambda$ is maximal. We illustrate this construction with two examples.

Example 3.4 The cocycle determined by a measured lamination $\lambda \in \mathcal{M}(\Lambda)$ is the tangent cocycle of the "ray" $\lambda_{t}=(1+t) \lambda \in \mathcal{M L}(S)$ at $t=0^{+}$. 
Example 3.5 Let $S$ be a punctured torus with meridian $\alpha$ and longitude $\beta$ (putting aside our assumption that $S$ is compact for a moment), and consider the family $\lambda_{t} \in \mathcal{M L}(S)$ defined by the conditions $\lambda_{t}(\alpha)=1$ and $\lambda_{t}(\beta)=t$. Thus for each $n \in \mathbb{N}$, the measured lamination $\lambda_{1 / n}$ is a simple closed curve with homology class $(n[\alpha]+[\beta])$ and weight $1 / n$.

The essential support of $\lambda_{t}$ at $t=0^{+}$is the geodesic lamination $\Lambda=\alpha \cup \eta$, where $\eta$ is an infinite simple geodesic that spirals toward $\alpha$ in each direction. The derivative $\dot{\lambda} \in \mathcal{H}(\Lambda, \mathbb{R})$ is a cocycle with full support and indefinite sign (compare Bonahon's example [5, page 104]).

This second example shows that the tangent cocycle may not be a transverse measure, and the essential support may not admit a measure of full support. This illustrates some of the difficulties of using differential methods on the space of measured laminations and of adapting the methods in Scannell-Wolf [36] to prove the main theorem.

Following Thurston, Bonahon showed that the association of a cocycle $\dot{\lambda}$ to a tangentiable family $\lambda_{t}$ provides a linear model for each $P L$ face of the tangent space $T_{\lambda_{0}} \mathcal{M L}(S)$ (see Bonahon [5] and Thurston [43, Section 6]). When $\operatorname{supp}\left(\lambda_{0}\right)$ is not maximal, however, no single maximal lamination $\Lambda$ can be chosen to contain the essential support of every family $\lambda_{t}$, and so there is no embedding $T_{\lambda_{0}} \mathcal{M L}(S) \rightarrow \mathcal{H}(\Lambda, \mathbb{R})$.

In contrast, when $\operatorname{supp}\left(\lambda_{0}\right)=\Lambda$ is maximal (a generic situation that excludes, for example, closed leaves), the tangent cocycle construction defines a homeomorphism $T_{\lambda_{0}} \mathcal{M L}(S) \simeq \mathcal{H}(\Lambda, \mathbb{R})$, giving the tangent space a canonical linear structure. This was observed in [43].

\section{Complex linearity}

So far we have seen real-valued cocycles on geodesic laminations arise in two different contexts: first as shearing cocycles providing coordinates for $\mathcal{T}(S)$, and then as tangent vectors to families of measured laminations. Building on these two constructions, the following result of Bonahon will allow us to connect the derivative of grafting with respect to $\mathcal{T}(S)$ and $\mathcal{M L}(S)$.

Theorem 3.6 (Bonahon [7, Proposition 5] and [4, Section 10]) Let $X \in \mathcal{T}(S)$ and $\lambda \in \mathcal{M L}(S)$. For each maximal geodesic lamination $\Lambda \in \mathcal{G} \mathcal{L}(S)$ containing the support of $\lambda$, there is a complex-linear map $L=L(\Lambda, \lambda, X): \mathcal{H}(\Lambda, \mathbb{C}) \rightarrow T_{\operatorname{Gr}_{\lambda} X} \mathcal{P}(S)$ which determines the tangent map of $\mathrm{Gr}$ in tangent directions carried by $\Lambda$, in the following sense. 
Let $\lambda_{t} \in \mathcal{M L}(S)$ be a tangentiable family of measured laminations with $\lambda_{0}=\lambda$ and with essential support contained in $\Lambda$, and let $\dot{\lambda}=\left.\frac{d}{d t}\right|_{t=0^{+}} \lambda_{t} \in \mathcal{H}(\Lambda, \mathbb{R})$. Let $X_{t} \in \mathcal{T}(S)$ be a smooth family of hyperbolic structures with $X_{0}=X$ whose derivative in the $\Lambda$ shearing embedding is $\dot{\sigma}=\left.\frac{d}{d t}\right|_{t=0^{+}} \sigma\left(X_{t}\right)$.

Then $t \mapsto \mathrm{Gr}_{\lambda_{t}} X_{t}$ is a tangentiable curve in $\mathcal{P}(S)$ and

$$
\left.\frac{d}{d t}\right|_{t=0^{+}} \mathrm{Gr}_{\lambda_{t}}=L(\dot{\sigma}+i \dot{\lambda}) .
$$

Similarly gr: $\mathcal{M L}(S) \times \mathcal{T}(S) \rightarrow \mathcal{T}(S)$ is tangentiable and its derivative has the same complex linearity property.

In terms of the piecewise linear structure of $\mathcal{M L}(S)$, Theorem 3.6 says that on each linear face of the tangent space $T_{(\lambda, X)}(\mathcal{M L}(S) \times \mathcal{T}(S))$, the tangent map $T_{(\lambda, X)} \mathrm{gr}$ is the restriction of a complex-linear map $\mathcal{H}(\Lambda, \mathbb{C}) \rightarrow T_{\operatorname{gr}_{\lambda} X} \mathcal{T}(S)$ (see [7, Section 2] and $[4$, Section 10]).

\section{Proof of the main theorem}

With the necessary background in place, we can now show that the tangent map of gr. $X$ has no kernel.

Proof of Theorem 2.6 Fix $X$ and suppose that $\left.\frac{d}{d t}\right|_{t=0^{+}} \operatorname{gr}_{\lambda_{t}} X=0$. Let $\dot{\lambda} \in \mathcal{H}(\Lambda, \mathbb{R})$ denote the derivative of $\lambda_{t}$ at $t=0^{+}$, with $\Lambda \in \mathcal{G} \mathcal{L}(S)$ maximal. We must show that $\dot{\lambda}=0$.

For all $t$ sufficiently small, the shearing $X_{t}=\operatorname{sh}_{t \dot{\lambda}} X \in \mathcal{T}(S)$ is defined and satisfies $X_{0}=X$ and $\dot{\lambda}=\frac{d}{d t} \sigma\left(X_{t}\right)$. Therefore by Theorem 3.6,

$$
i\left[\left.\frac{d}{d t}\right|_{t=0^{+}} \operatorname{gr}_{\lambda_{0}} X_{t}\right]=i L(\dot{\lambda})=L(i \dot{\lambda})=\left.\frac{d}{d t}\right|_{t=0^{+}} \operatorname{gr}_{\lambda_{t}} X_{0}=0,
$$

where $L$ is the complex-linear map representing the tangent map of gr in tangent directions carried by $\Lambda$.

By Theorem 1.2, the map $\operatorname{gr}_{\lambda_{0}}$ is an immersion, and so $\left.\frac{d}{d t}\right|_{t=0^{+}} X_{t}=0$. Thus

$$
\dot{\lambda}=\frac{d}{d t} \sigma\left(X_{t}\right)=0 \text {. }
$$

If the grafting map were continuously differentiable in the usual sense, the proof of the main theorem would now be straightforward, using linearity of the derivative and the inverse function theorem to conclude that gr. $X$ is a local diffeomorphism. We will 
follow this general outline, but we will need to use additional properties of the grafting maps to strengthen the infinitesimal result to a local one.

In fact, some argument specific to grafting is necessary at this point. In general, a tangent map that has no kernel need not be injective (consider $\mathbb{C} \rightarrow \mathbb{R}^{+}$by $z \mapsto|z|$ at $z=0$ ), and even if the tangent map is injective, tangentiability does not imply the continuous variation of derivatives needed for the inverse function theorem.

With these potential problems in mind, we analyze the $X$-grafting map gr. $X$ as the composition of the projective $X$-grafting map Gr. $X$ and the smooth projection $\pi: \mathcal{P}(S) \rightarrow \mathcal{T}(S)$. Let $N=\operatorname{dim} \mathcal{T}(S)=\operatorname{dim} \mathcal{M L}(S)=\frac{1}{2} \operatorname{dim} \mathcal{P}(S)=6 g-6$.

Let $G_{X}=\left\{\mathrm{Gr}_{\lambda} X \mid \lambda \in \mathcal{M L}(S)\right\} \subset \mathcal{P}(S)$ denote the image of Gr. $X$. Recall the map $p_{\mathcal{T}}: \mathcal{P}(S) \rightarrow \mathcal{T}(S)$ is defined by $p_{\mathcal{T}}\left(\operatorname{Gr}_{\lambda} X\right)=X$, and so $G_{X}=p_{\mathcal{T}}^{-1}(X)$ is a fiber of this map. Since $p_{\mathcal{T}}$ is a $C^{1}$ submersion (by Corollary 2.5), the set $G_{X}$ is actually a $C^{1}$ submanifold of $\mathcal{P}(S)$ of dimension $N=\operatorname{dim} \mathcal{P}(S)-\operatorname{dim} \mathcal{T}(S)$. In particular, $G_{X}$ is smoother than its tangentiable parameterization by $\mathcal{M L}(S)$ would suggest.

We can recast Theorem 2.6 as a result about the tangent space to $G_{X}$ as follows.

Theorem 4.1 For any $X \in \mathcal{T}(S)$, the $C^{1}$ submanifold $G_{X} \subset \mathcal{P}(S)$ is transverse to the map $\pi: \mathcal{P}(S) \rightarrow \mathcal{T}(S)$; that is, for any $Z \in G_{X}$, we have $T_{Z} G_{X} \cap(\operatorname{ker} d \pi)=\{0\}$. Equivalently, the distributions $\operatorname{ker} d \pi$ and $\operatorname{ker} d p_{\mathcal{T}}$ in $T \mathcal{P}(S)$ are transverse.

Proof Suppose not, ie that there exists $v \in\left(T_{Z} G_{X} \cap \operatorname{ker} d \pi\right)$ with $v \neq 0$. Since $Z \in G_{X}$, we have $Z=\operatorname{Gr}_{\lambda} X$ for some $\lambda \in \mathcal{M L}(S)$. Choose a $C^{1}$ path $Z_{t}$ in $G_{X}$ with $Z_{0}=Z$ and $\left.\frac{d}{d t}\right|_{t=0^{+}} Z_{t}=v$, and let $\mathrm{Gr}^{-1}\left(Z_{t}\right)=\left(\lambda_{t}, X\right)$. Since $\mathrm{Gr}^{-1}$ is a bitangentiable homeomorphism, the family $\lambda_{t}$ is tangentiable and satisfies $\left.\frac{d}{d t}\right|_{t=0^{+}} \lambda_{t} \neq 0$. On the other hand, $\left.\frac{d}{d t}\right|_{t=0^{+}} \operatorname{gr}_{\lambda_{t}} X=\left.\frac{d}{d t}\right|_{t=0^{+}} \pi\left(Z_{t}\right)=d \pi\left(\left.\frac{d}{d t}\right|_{t=0^{+}} Z_{t}\right)=0$, contradicting Theorem 2.6.

While a general tangent map can have no kernel and yet fail to be injective, the fact that $G_{X}$ is $C^{1}$ (and thus has linear tangent spaces) rules out this behavior for Gr. $X$.

Theorem 4.2 The tangent map $T_{\lambda}$ gr. $X$ of the conformal $X$-grafting map is a homeomorphism.

Proof We study the diagram of grafting maps:

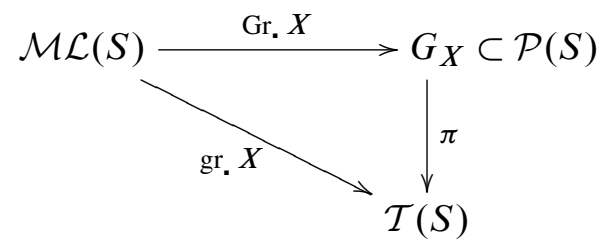


Fix $\lambda \in \mathcal{M L}(S)$ and for brevity let $f=T_{\lambda}$ gr. $X$ be the tangent map of the conformal $X$-grafting map; our goal is to show that $f$ is a homeomorphism.

Similarly, let $F=T_{\lambda}$ Gr. $X$ be the tangent map of the projective $X$-grafting map. Then $f=d \pi \circ F$, where $d \pi$ is the differential of $\pi: \mathcal{P}(S) \rightarrow \mathcal{T}(S)$ at $Z=\operatorname{Gr}_{\lambda} X$, ie the tangent maps form a corresponding diagram:

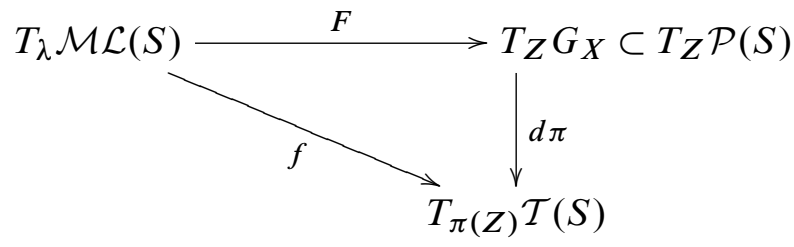

Note that $F$ and $f$ are continuous homogeneous maps, while $d \pi$ is linear.

Since $\mathrm{Gr}$ is a tangentiable homeomorphism, its restriction Gr. $X: \mathcal{M L}(S) \rightarrow \mathcal{P}(S)$ is a tangentiable injection, ie its tangent map $F$ is both injective and a homogeneous homeomorphism onto its image. Since $G_{X}$ is a $C^{1}$ submanifold of $\mathcal{P}(S)$, the image of $F$ is the linear subspace $T_{Z} G_{X} \subset T_{Z} \mathcal{P}(S)$.

First we show that $f$ injective. Suppose on the contrary there exist $\dot{\lambda}_{1}, \dot{\lambda}_{2} \in T_{\lambda} \mathcal{M L}(S)$, distinct and nonzero, and $f\left(\dot{\lambda}_{1}\right)=f\left(\dot{\lambda}_{2}\right)$. Then $v=F\left(\dot{\lambda}_{1}\right)-F\left(\dot{\lambda}_{2}\right) \in T_{Z} G_{X}$ is nonzero since $F$ is injective, and so $v \in \operatorname{ker} d \pi$, which contradicts Theorem 4.1.

Thus $f: T_{\lambda} \mathcal{M L}(S) \rightarrow T_{\operatorname{gr}_{\lambda} X} \mathcal{T}(S)$ is injective. Since $f$ is also a homogeneous map between cones of the same dimension, it is a homeomorphism.

Remark The fact that $T_{\lambda}$ Gr. $X$ is a homeomorphism onto a linear subspace of $T_{\mathrm{Gr}_{\lambda} X} \mathcal{P}(S)$ is also observed by Bonahon in [7, proof of Proposition 12].

We complete our study of Gr. $X$ and gr. $X$ by proving the main theorem.

Proof of Theorem 1.1 Let us consider the restriction of the forgetful map $\pi: \mathcal{P}(S) \rightarrow$ $\mathcal{T}(S)$ to the $C^{1}$ submanifold $G_{X} \subset \mathcal{P}(S)$. Since $G_{X}$ is $N$-dimensional, this projection is a local diffeomorphism at $Z \in G_{X}$ if the subspaces $T_{Z} G_{X}$ and ker $d \pi$ of $\mathcal{T}_{Z} \mathcal{P}(S)$ are transverse (by the inverse function theorem). By Theorem 4.1, this is true for every $Z \in G_{X}$, so $\left.\pi\right|_{G_{X}}$ is a local $C^{1}$ diffeomorphism.

Thus the conformal $X$-grafting map is the composition of the homeomorphism Gr. $X: \mathcal{M L}(S) \rightarrow G_{X}$ and the local homeomorphism $\left.\pi\right|_{G_{X}}: G_{X} \rightarrow \mathcal{T}(S)$, so gr. $X$ is a local homeomorphism. As we noted in Section 2, by the properness of gr. $X$ (Theorem 2.1), it follows that this map is a homeomorphism. Thus $X$-grafting is a tangentiable homeomorphism with injective tangent maps (Theorem 4.2), which by Lemma 2.2 is a bitangentiable homeomorphism. 


\section{Applications}

In this section, we collect some applications of the main theorem itself and of the techniques used in its proof, and we discuss some related questions about grafting coordinates and $\mathbb{C} \mathbb{P}^{1}$ structures.

\section{Projections}

We begin by proving the main corollary of Theorem 1.1 and Theorem 1.2 about the grafting coordinates for a fiber $P(X)=\pi^{-1}(X) \subset \mathcal{P}(S)$.

Corollary 1.3 For each $X \in \mathcal{T}(S)$, the space $P(X)$ of $\mathbb{C P}^{1}$ structures on $X$ is a graph over each factor in the grafting coordinate system. In fact, we have:

(1) The projection $\left.p_{\mathcal{T}}\right|_{P(X)}: P(X) \rightarrow \mathcal{T}(S)$ is a $C^{1}$ diffeomorphism.

(2) The projection $\left.p_{\mathcal{M L}}\right|_{P(X)}: P(X) \rightarrow \mathcal{M L}(S)$ is a bitangentiable homeomorphism.

Proof First we consider the regularity of the maps. Bonahon showed that $p_{\mathcal{T}}$ is $C^{1}$ (Theorem 2.4), and $p_{\mathcal{M L}}$ is tangentiable because it is the composition of $\mathrm{Gr}^{-1}$, a bitangentiable homeomorphism (Theorem 2.3), and the projection to one factor of a product of tangentiable manifolds.

(1) First of all, the map $\left.p_{\mathcal{T}}\right|_{P(X)}$ is a homeomorphism because it has inverse map

$$
Y \mapsto \mathrm{Gr}_{(\mathrm{gr} . X)^{-1}(Y)} Y
$$

where the map $(\operatorname{gr} . X)^{-1}: \mathcal{T}(S) \rightarrow \mathcal{M L}(S)$ exists by Theorem 1.1.

Thus it suffices to show that $\left.p_{\mathcal{T}}\right|_{P(X)}$ is a local diffeomorphism. But the kernel of $\left.d p_{\mathcal{T}}\right|_{P(X)}$ is the intersection of $\operatorname{ker} d \pi$ and $\operatorname{ker} d p_{\mathcal{T}}$ in $T \mathcal{P}(S)$, which is zero by Theorem 4.1. So the derivative of $\left.p_{\mathcal{T}}\right|_{P(X)}$ is an isomorphism, and by the inverse function theorem this map is a local diffeomorphism.

(2) As in (1), we first show that $\left.p_{\mathcal{M L}}\right|_{P(X)}$ is a homeomorphism by exhibiting an inverse map,

$$
\lambda \mapsto \operatorname{Gr}_{\lambda}\left(\operatorname{gr}_{\lambda}^{-1}(Y)\right)
$$

where $\operatorname{gr}_{\lambda}^{-1}$ exists by Theorem 1.2.

By Lemma 2.2, we need only show that the tangent map of $\left.p_{\mathcal{M L}}\right|_{P(X)}$ is everywhere injective. That this tangent map has no kernel also follows easily from Theorem 1.2, 
but to show injectivity we will use an argument modeled on the proofs of Theorem 2.6 and Theorem 4.2.

Suppose on the contrary that two distinct, nonzero tangent vectors $v_{1}, v_{2} \in T_{Z} P(X)$ have the same image in $T_{\lambda} \mathcal{M L}(S)$ under the tangent map of $p_{\mathcal{M L}}$, where $Z=\mathrm{Gr}_{\lambda} Y$. Then differentiable paths in $P(X)$ with tangent vectors $v_{1}$ and $v_{2}$ are mapped by $\mathrm{Gr}^{-1}$ to tangentiable paths $\mathrm{Gr}_{\lambda_{t}^{(1)}} Y_{t}^{(1)}$ and $\mathrm{Gr}_{\lambda_{t}^{(2)}} Y_{t}^{(2)}$, respectively, where $Y_{0}^{(k)}=Y$ and $\lambda_{0}^{(k)}=\lambda$ for $k=1,2$.

In this notation, the image of $v_{k}$ by the tangent map $T_{Z} p_{\mathcal{M L}}$ is $\left.\frac{d}{d t}\right|_{t=0^{+}} \lambda_{t}^{(k)}$, so we have $\left.\frac{d}{d t}\right|_{t=0^{+}} \lambda_{t}^{(1)}=\left.\frac{d}{d t}\right|_{t=0^{+}} \lambda_{t}^{(2)}=\dot{\lambda}$. In particular there is a single geodesic lamination $\Lambda$ containing the essential support of both families $\lambda_{t}^{(k)}$, and their common tangent vector defines a cocycle $\dot{\lambda} \in \mathcal{H}(\Lambda, \mathbb{R})$. Using the shearing embedding of Teichmüller space in $\mathcal{H}(\Lambda, \mathbb{R})$ gives cocycles $\dot{\sigma}_{k}=\left.\frac{d}{d t}\right|_{t=0^{+}}\left(\sigma^{\Lambda}\left(Y_{t}^{(k)}\right)\right)$, and since $v_{1} \neq v_{2}$ we have $\dot{\sigma}_{1} \neq \dot{\sigma}_{2}$.

By Theorem 3.6, there is a complex-linear map $L: \mathcal{H}(\Lambda, \mathbb{C}) \rightarrow T_{X} \mathcal{T}(S)$ that gives the tangent map of $\mathrm{Gr}$ for tangent vectors to $\mathcal{M L}(S) \times \mathcal{T}(S)$ at $(\lambda, X)$ representable by complex-valued cocycles on $\Lambda$, so $v_{k}=L\left(\dot{\sigma}_{k}+i \dot{\lambda}\right)$. Now consider $\dot{\sigma}=\dot{\sigma}_{1}-\dot{\sigma}_{2} \neq 0$, which is the tangent vector to the shearing family $Y_{t}=\operatorname{sh}_{t \dot{\sigma}} Y$; we have

$$
\begin{aligned}
\left.\frac{d}{d t}\right|_{t=0^{+}} \operatorname{Gr}_{\lambda} Y_{t} & =L(\dot{\sigma}) \\
& =L\left(\left(\dot{\sigma}_{1}+i \dot{\lambda}\right)-\left(\dot{\sigma}_{2}+i \dot{\lambda}\right)\right) \\
& =v_{1}-v_{2} \in T_{Z} P(X),
\end{aligned}
$$

hence $\left.\frac{d}{d t}\right|_{t=0^{+}} \operatorname{gr}_{\lambda} Y_{t}=0$, which by Theorem 1.2 implies that $\left.\frac{d}{d t}\right|_{t=0^{+}} Y_{t}=0$, and so $\dot{\sigma}=\left.\frac{d}{d t}\right|_{t=0^{+}} \sigma^{\Lambda}\left(Y_{t}\right)=0$, a contradiction. Thus the tangent map of $p_{\mathcal{M L}}$ is injective, as required.

Remark Since Bonahon constructs an explicit example to show that the full projection map $p_{\mathcal{T}}: \mathcal{P}(S) \rightarrow \mathcal{T}(S)$ is not necessarily $C^{2}$, it would be interesting to know if a similar construction could be used to show that $\left.p_{\mathcal{T}}\right|_{P(X)}$ need not be $C^{2}$.

\section{Pruning}

Since the $\lambda$-grafting map $\operatorname{gr}_{\lambda}: \mathcal{T}(S) \rightarrow \mathcal{T}(S)$ is a homeomorphism (Theorem 1.2), there is an inverse map $\operatorname{pr}_{\lambda}: \mathcal{T}(S) \rightarrow \mathcal{T}(S)$ which we call pruning by $\lambda$. Roughly speaking, grafting by $\lambda$ inserts a Euclidean subsurface along the leaves of $\lambda$, and pruning by $\lambda$ removes it. Allowing $\lambda$ to vary, we obtain the pruning map pr: $\mathcal{M L}(S) \times \mathcal{T}(S) \rightarrow$ $\mathcal{T}(S)$, and fixing $X$ we have the $X$-pruning map pr. $X: \mathcal{M L}(S) \times\{X\} \rightarrow \mathcal{T}(S)$.

We can reformulate Corollary 1.3 in terms of pruning as follows. 


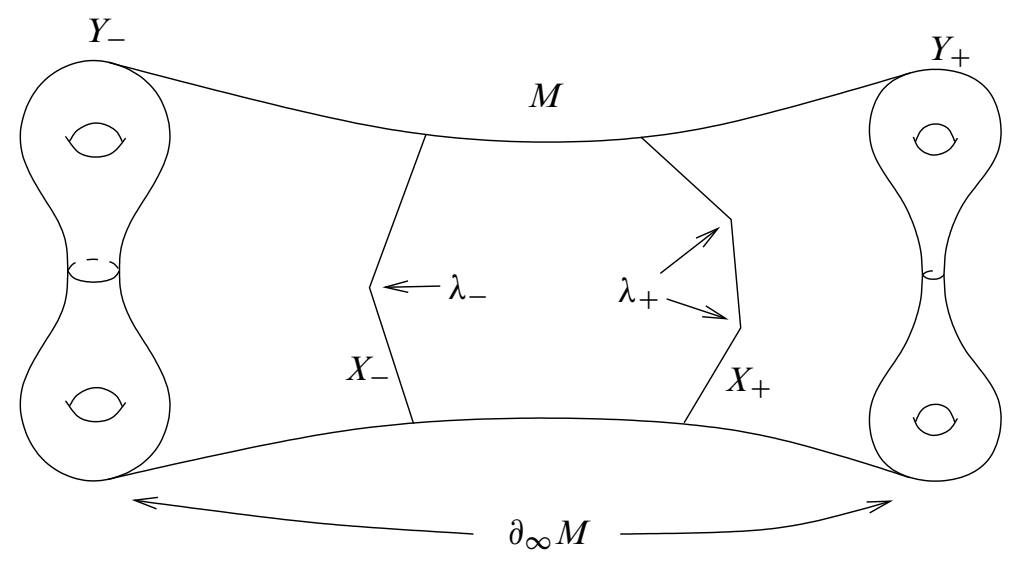

Figure 3: Geometric data associated to a quasi-Fuchsian manifold $M \in \mathcal{Q F}$. In a Bers slice, $Y_{-}$is fixed.

Corollary 5.1 For each $X \in \mathcal{T}(S)$, the $X$-pruning map pr. $X: \mathcal{M L}(S) \rightarrow \mathcal{T}(S)$ is a bitangentiable homeomorphism.

Proof The graph of the $X$-pruning map consists of the pairs $(\lambda, Y)$ such that $\operatorname{gr}_{\lambda} Y=$ $X$, which is simply the fiber $P(X) \subset \mathcal{P}(S) \simeq \mathcal{M L}(S) \times \mathcal{T}(S)$. In terms of the projections projections $p_{\mathcal{M L}}: P(X) \rightarrow \mathcal{M L}(S)$ and $p_{\mathcal{T}}: P(X) \rightarrow \mathcal{T}(S)$, we have pr. $X=p_{\mathcal{T}} \circ p_{\mathcal{M L}}^{-1}$, which is a bitangentiable homeomorphism by Corollary 1.3.

Previously it was known that pr. $X$ is a "rough homeomorphism", ie a proper map of degree 1, and that is has a natural extension to the Thurston compactification of $\mathcal{T}(S)$ and the projective compactification of $\mathcal{M L}(S)$ by $\mathbb{P} \mathcal{M L}(S)=(\mathcal{M L}(S)-\{0\}) / \mathbb{R}^{+}$. Furthermore, the resulting boundary map $\mathbb{P} \mathcal{M L}(S) \rightarrow \mathbb{P} \mathcal{M L}(S)$ is the antipodal map relative to $X$. For details, see Dumas [11].

\section{Internal coordinates for the Bers slice}

Let $\mathcal{Q F}=\mathcal{Q F}(S)$ denote the space of marked quasi-Fuchsian hyperbolic 3-manifolds homeomorphic to $S \times \mathbb{R}$ (see Bers [1] and Nag [30]). Each such manifold $M \in \mathcal{Q F}$ has ideal boundary $\partial_{\infty} M=Y_{+} \sqcup Y_{-}$with conformal structures $Y_{ \pm} \in \mathcal{T}(S)$ and convex core boundary surfaces $X_{ \pm} \in \mathcal{T}(S)$ with bending laminations $\lambda_{ \pm} \in \mathcal{M L}(S)$ (represented schematically in Figure 3). Furthermore, the convex core and ideal boundary surfaces satisfy gr $_{\lambda_{ \pm}} X_{ \pm}=Y_{ \pm}$(McMullen [29, Theorem 2.8], see also Kamishima-Tan [20]). In his celebrated holomorphic embedding [2] of the Teichmüller space $\mathcal{T}(S)$ into $\mathbb{C}^{3 g-3}$, Bers focused on the slice $\mathcal{B}_{Y} \subset \mathcal{Q F}$ of those quasi-Fuchsian manifolds with a 
fixed ideal boundary surface $Y_{-}=Y$. Bers showed that a quasi-Fuchsian manifold $M \in \mathcal{B}_{Y}$ is uniquely determined by its other conformal boundary surface $Y_{+} \in \mathcal{T}(S)$, which can be chosen arbitrarily (the Simultaneous Uniformization Theorem; see Bers [1]).

A corollary of our main theorem is that $\mathcal{B}_{Y}$ may also be parameterized by the hyperbolic structure $X_{-}$.

Corollary 5.2 Let $M, M^{\prime} \in \mathcal{Q F}$ be marked quasi-Fuchsian manifolds, and suppose an end of $M$ (respectively $M^{\prime}$ ) has ideal boundary $Y$ (resp. $Y^{\prime}$ ) and the associated convex core boundary surface has hyperbolic metric $X$ (resp. $X^{\prime}$ ). If $Y$ and $Y^{\prime}$ are conformally equivalent and $X$ and $X^{\prime}$ are isometric, then $M$ is isometric to $M^{\prime}$.

Proof By hypothesis $Y^{\prime}=Y$ and $X^{\prime}=X$ as points in Teichmüller space, so the bending measures $\lambda$ and $\lambda^{\prime}$ of the convex core boundaries satisfy $\operatorname{gr}_{\lambda} X=\operatorname{gr}_{\lambda^{\prime}} X=Y$. By Theorem 1.1, we have $\lambda=\lambda^{\prime}$.

A quasi-Fuchsian manifold $M$ is uniquely determined up to isometry by the hyperbolic metric $X$ and bending lamination $\lambda$ of one of its convex core boundary surfaces, since one can use $X$ and $\lambda$ to construct the associated equivariant pleated plane in $\mathbb{H}^{3}$ and its holonomy group $\pi_{1} M \subset \mathrm{PSL}_{2}(\mathbb{C})$ (see Epstein-Marden [13]). As $M$ and $M^{\prime}$ share these data, they are isometric.

For another perspective on this corollary, we can consider the Bers slice $\mathcal{B}_{Y}$ as a subset of $P(Y)$, where a quasi-Fuchsian manifold $M \in \mathcal{B}_{Y}$ is identified with the projective structure it induces on its ideal boundary surface $Y$. Then Corollary 5.2 is equivalent to the statement that for every $Y \in \mathcal{T}(S)$, the projection map $\left.p_{\mathcal{T}}\right|_{\mathcal{B}_{Y}}: \mathcal{B}_{Y} \rightarrow \mathcal{T}(S)$ is injective. Of course this can also be derived from Corollary 1.3, which shows, furthermore, that $\left.p_{T}\right|_{\mathcal{B}_{Y}}$ is a $C^{1}$ embedding (since $\mathcal{B}_{Y} \subset P(Y)$ is open).

\section{Remarks}

(1) In [36], it was observed that a manifold $M \in \mathcal{B}_{Y}$ is also determined by the bending lamination $\lambda_{-} \in \mathcal{M L}(S)$ on the same side as the fixed conformal structure $Y$. It is not known whether either the bending lamination $\lambda_{+}$or the hyperbolic structure $X_{+}$determine elements of $\mathcal{B}_{Y}$.

(2) More generally, one can ask what geometric data determine $M \in \mathcal{Q F}$ up to isometry. Bonahon and Otal [9] showed that if $\lambda_{+}$and $\lambda_{-}$bind the surface and are supported on simple closed curves, then the pair $\left(\lambda_{+}, \lambda_{-}\right)$determines $M \in \mathcal{Q F}$ uniquely. Recently, Bonahon [8] showed that this restriction to simple closed curves may be lifted for elements of $\mathcal{Q F}$ which are sufficiently close to the Fuchsian subspace $\mathcal{F} \subset \mathcal{Q F}$, and Series [39] proved that $\lambda_{+}$and $\lambda_{-}$ determine $M$ when $S$ is a once-punctured torus. 


\section{Grafting coordinates and rays}

In this final section we discuss how the ray structure of $\mathcal{M L}(S)$ is transported to $\mathcal{T}(S)$ by the $X$-grafting map.

Recall (from Section 2) that the action of $\mathbb{R}^{+}$on $\mathcal{M L}(S)$ by scaling transverse measures gives this space the structure of a cone, with the empty lamination 0 as its base point. By Theorem 1.1, for each $X \in \mathcal{T}(S)$ we can use the $X$-grafting map to parameterize the Teichmüller space by $\mathcal{M L}(S)$, providing a global system of "polar coordinates" centered at $X$. In this coordinate system, the ray $\mathbb{R}^{+} \lambda \in \mathcal{M L}(S)$ corresponds to the grafting ray $\left\{\operatorname{gr}_{t \lambda} X \mid t \in \mathbb{R}^{+}\right\}$, a properly embedded path starting at $X$, and Teichmüller space is the union of these rays.

It would be interesting to understand the geometry of this coordinate system, and especially the grafting rays. Thus we ask the following question.

Question What is the behavior of the grafting ray $t \mapsto \operatorname{gr}_{t \gamma} X$ and how does it depend on $\lambda$ and $X$ ?

Theorem 6.2 and Theorem 6.6 below address this question by estimating the extremal and hyperbolic lengths of the grafting lamination (and their derivatives) along a ray. The asymptotic behavior of certain grafting rays in relation to the Teichmüller metric has also been investigated by Díaz and Kim, see [10].

Naturally, one first wonders about the regularity of grafting rays, since the grafting map itself exhibits a combination of tangentiable and differentiable behavior. However, along rays the grafting map is as smooth as possible.

Theorem 6.1 (McMullen [29]) The grafting ray $t \mapsto \operatorname{gr}_{t \lambda}$ is a real-analytic map from $\mathbb{R}^{+}$to $\mathcal{T}(S)$; in fact, it is the restriction of a real-analytic map $\{t \geq-\epsilon\} \rightarrow \mathcal{T}(S)$ for some $\epsilon>0$ (depending on $\lambda$ ). Furthermore, if $\lambda_{n} \rightarrow \lambda$ in $\mathcal{M L}(S)$, then the $\lambda_{n}$ grafting rays converge in $C^{\omega}$ to the $\lambda$ grafting ray.

\section{Remarks}

(1) In [29] it is shown that for any $\lambda \in \mathcal{M L}(S)$, the complex earthquake map $\mathrm{eq}_{\lambda}: \mathbb{H} \rightarrow \mathcal{T}(S)$ is holomorphic and extends to an open neighborhood of $\mathbb{H} \cup \mathbb{R}$ [29, Proposition 2.6 and Theorem 2.10]. Furthermore these maps vary continuously with $\lambda \in \mathcal{M L}(S)$ [29, Theorem 2.5], as do their derivatives, since they are holomorphic. Since the grafting ray is the restriction of the complex earthquake to $i \mathbb{R}$, Theorem 6.1 follows immediately. 
(2) The regularity of grafting rays and complex earthquakes is closely related to (and in part, an application of) the analyticity of quake-bend deformations of surface group representations in $\mathrm{PSL}_{2}(\mathbb{C})$ established by Epstein and Marden [13].

\section{Extremal length}

When $\lambda$ is supported on a simple closed geodesic $\gamma$, it is clear that a large grafting will result in a surface in which $\gamma$ has small extremal length, because $\operatorname{gr}_{t \gamma} X$ contains an annulus of large modulus homotopic to $\gamma$. Refining this intuition, we will establish the estimate.

Theorem 6.2 For each $X \in \mathcal{T}(S)$, the extremal length of $\lambda$ is of order $1 / t$ on the $\lambda$-grafting ray $t \mapsto \operatorname{gr}_{t \lambda} X$ and is monotone decreasing for all $t \gg 0$. Specifically, we have

(1) $E\left(\lambda, \operatorname{gr}_{t \lambda} X\right)=\frac{\ell(\lambda, X)}{t}+O\left(t^{-2}\right)$, and

(2) $\frac{d}{d t} E\left(\lambda, \operatorname{gr}_{t \lambda} X\right)=\frac{-\ell(\lambda, X)}{t^{2}}+O\left(t^{-3}\right)$

where $E(\lambda, Y)$ denotes the extremal length of $\lambda$ on the Riemann surface $Y$. The implicit constants depend only on $\chi(S)$.

Note that Theorem 6.2 includes the results stated in the introduction as Theorem 1.4. Before giving the proof of Theorem 6.2, we fix notation and recall some concepts from Teichmüller theory used therein.

\section{Annuli, Jenkins-Strebel differentials, and foliations}

Let $A$ be an annular Riemann surface of modulus $M$ and let $E(A)=1 / M=E(\gamma, A)$ be the extremal length of $\gamma$, the nontrivial isotopy class of simple closed curves on $A$. Then $A$ is isomorphic to a rectangle $R_{A}=[0, E(A)] \times(0,1) \subset \mathbb{C}$ with its vertical sides identified. We call the complex local coordinate $z$ on $A$ coming from this realization the natural coordinate for $A$. Similarly, the induced flat metric $|d z|$ on $A$ is the natural metric, with respect to which $A$ is a Euclidean cylinder of height 1 and circumference $E(A)$.

For any isotopy class $\gamma$ of simple closed curves on a compact Riemann surface $X$, there is a unique embedded annulus $A_{\gamma} \subset X$ homotopic to $\gamma$ of maximum modulus $\operatorname{Mod}\left(A_{\gamma}\right)=1 / E(\gamma, X)$. The annulus $A_{\gamma}$ is dense in $Y$, and if $z$ is the natural coordinate for $A_{\gamma}$, the quadratic differential $d z^{2}$ on $A$ extends holomorphically to a quadratic differential on $X$, the Jenkins-Strebel differential for $\gamma$. 
A holomorphic quadratic differential $\phi$ on a Riemann surface $X$ has an associated singular horizontal foliation $\mathcal{F}(\phi)$ whose leaves integrate the distribution of tangent vectors $v$ satisfying $\phi(v) \geq 0$. Integration of $|\operatorname{Im} \sqrt{\phi}|$ gives a transverse measure on $\mathcal{F}(\phi)$. Similarly $\mathcal{F}(-\phi)$ is the vertical foliation, whose transverse measure comes from $|\operatorname{Re} \sqrt{\phi}|$.

When $\phi$ is a Jenkins-Strebel differential on a compact surface, the nonsingular leaves of $\mathcal{F}(\phi)$ are closed and homotopic to $\gamma$; in the realization of the Jenkins-Strebel annulus $A_{\gamma}$ as a rectangle with identifications, these are the horizontal lines, while leaves of $\mathcal{F}(-\phi)$ are vertical lines. The transverse measures for $\mathcal{F}(\phi)$ and $\mathcal{F}(-\phi)$ are given by $|d y|$ and $|d x|$ in the rectangle, respectively. Thus the closed leaves of $\mathcal{F}(\phi)$ have total measure $E\left(A_{\gamma}\right)=E(\gamma, X)$ with respect to the transverse measure of the vertical foliation $\mathcal{F}(-\phi)$. Furthermore these closed leaves realize the minimum transverse measure among all curves homotopic to $\gamma$ (see Gardiner [16, Lemma 11.5.3]).

\section{Pairing and extremal length}

The natural pairing between Beltrami differentials $\mu=\mu(z) d \bar{z} / d z$ (with $\mu(z) \in L^{\infty}$ ) and integrable holomorphic quadratic differentials $\phi=\phi(z) d z^{2}$ on a Riemann surface $X$ is given by

$$
\langle\mu, \phi\rangle=\operatorname{Re} \int_{X} \mu \phi=\int_{X} \mu(z) \phi(z)|d z|^{2} .
$$

When $X=A$ is an annulus with natural coordinate $z_{A}$, pairing a Beltrami differential with $\phi=d z_{A}^{2}$ gives the infinitesimal change in extremal length $E(A)$ (see [16, Section 1.9]); that is, if $A_{t}$ is a family of annuli identified by a family of quasiconformal maps with derivative $\mu_{t}$, then

$$
\frac{d}{d t} E\left(A_{t}\right)=2\left\langle\mu(t), d z_{A_{t}}^{2}\right\rangle .
$$

Similarly, when $\phi$ is a Jenkins-Strebel differential on a compact surface $X$, pairing with $\phi$ gives the differential of the extremal length function on Teichmüller space.

Theorem 6.3 (Gardiner [16, Theorem 11.8.5]) Let $t \mapsto X_{t} \in \mathcal{T}(S)$ be a differentiable path whose tangent vector is represented by the Beltrami differential $\mu(t)$ on $X_{t}$. Let $\gamma$ be an isotopy class of simple closed curves and $E(t)=E\left(\gamma, X_{t}\right)$ its extremal length on $X_{t}$. Then

$$
E^{\prime}(t)=2\langle\mu(t), \phi(t)\rangle
$$

where $\phi(t)$ is the Jenkins-Strebel differential for $\gamma$ on $X_{t}$. 


\section{Stretching annuli}

Let $X$ be a compact Riemann surface and $A \subset X$ an annulus in the homotopy class of $\gamma$, a simple closed curve. The natural coordinate $z$ on $A$ gives a Beltrami differential $d \bar{z} / d z$ on $A$, which extends to a Beltrami differential on $X$ by setting it to zero on $(X-A)$. This differential represents an infinitesimal affine stretch of $A$. We will be interested in the extent to which $d \bar{z} / d z$ affects the extremal length of $\gamma$, as estimated in the following Lemma.

Lemma 6.4 Let $A \subset X, \gamma$, and $z$ be as above, and let $\phi$ be the Jenkins-Strebel differential on $X$ for $\gamma$. Then

$$
E(X) \geq\left\langle\frac{d \bar{z}}{d z}, \phi\right\rangle \geq \frac{2 E(X)^{2}}{E(A)}-E(X)
$$

where $E(A)=E(\gamma, A)$ and $E(X)=E(\gamma, X)$ are the extremal lengths of $\gamma$ on these two surfaces.

\section{Remarks}

(1) When $A=A_{\gamma}$ is the Jenkins-Strebel annulus (of maximum modulus), we have $E(A)=E(X)$ and both inequalities in Lemma 6.4 become equalities. However this is clear since $d \bar{z} / d z=\bar{\phi} /|\phi|$ if $z$ is the natural coordinate of the JenkinsStrebel annulus. The point of the Lemma is that we also have tight bounds for the pairing when $A$ has nearly maximum modulus.

(2) A related estimate for nearly maximal annuli is used in Kerckhoff's proof of that that foliation map $\mathcal{F}: Q(X) \rightarrow \mathcal{M L}(S)$ is a homeomorphism, see [22, Lemma 3.2].

Proof Throughout the proof we use the natural coordinate $z$ to identify $A$ with a rectangle $R_{A} \subset \mathbb{C}$ whose vertical sides are identified.

Writing the restriction of $\phi$ to $A$ in terms of the natural coordinate, we have

$$
\phi=\phi(z) d z^{2}=(\alpha+i \beta)^{2} d z^{2},
$$

where $\alpha$ and $\beta$ are real-valued functions defined locally up to a common sign away from the zeros of $\phi$; in particular the functions $\alpha^{2}, \beta^{2}$ and $|\alpha|$ are well-defined almost 
everywhere. We want to estimate

$$
\begin{aligned}
\left\langle\frac{d \bar{z}}{d z}, \phi\right\rangle & =\operatorname{Re} \int_{A} \frac{d \bar{z}}{d z} \phi(z) d z^{2} \\
& =\int_{A} \operatorname{Re}\left((\alpha+i \beta)^{2}\right)|d z|^{2} \\
& =\int_{0}^{1} \int_{0}^{E(A)}\left(\alpha^{2}-\beta^{2}\right) d x d y .
\end{aligned}
$$

Note that the pairing is computed as an integral over $A$, rather than $X$, because the Beltrami coefficient $d \bar{z} / d z$ is understood to be zero on $(X-A)$.

We first derive the upper bound on the pairing. Since $A$ is a subset of $X$, we have

$$
\int_{0}^{1} \int_{0}^{E(A)}\left(\alpha^{2}+\beta^{2}\right) d x d y=\int_{A}|\phi| \leq \int_{X}|\phi|=E(X) .
$$

Since $\beta^{2} \geq 0$, the same upper bound applies to the integral of $\alpha^{2}-\beta^{2}$, giving

$$
\left\langle\frac{d \bar{z}}{d z}, \phi\right\rangle=\int_{0}^{1} \int_{0}^{E(A)}\left(\alpha^{2}-\beta^{2}\right) d x d y \leq E(X) .
$$

To establish the lower bound on the pairing, note that the horizontal lines in $R_{A}$ represent closed curves in $X$ homotopic to $\gamma$, so the total transverse measure of any one of these with respect to $\mathcal{F}(-\phi)$ is at least $E(X)$. The transverse measure of a curve is the integral of $|\operatorname{Re} \sqrt{\phi}|=|\alpha d x-\beta d y|$, but $d y=0$ on horizontal lines, so we have

$$
\int_{0}^{E(A)}|\alpha(x+i y)| d x \geq E(X)
$$

for all $y \in[0,1]$. Integrating over $y$ and applying the Cauchy-Schwarz inequality, we obtain

$$
\int_{0}^{1} \int_{0}^{E(A)} \alpha^{2} d x d y \geq \frac{E(X)^{2}}{E(A)}
$$

Multiplying (6-3) by 2 and subtracting (6-2) we have

$$
\left\langle\frac{d \bar{z}}{d z}, \phi\right\rangle=\int_{0}^{1} \int_{0}^{E(A)}\left(\alpha^{2}-\beta^{2}\right) d x d y \geq \frac{2 E(X)^{2}}{E(A)}-E(X) .
$$

Note that the proof of Lemma 6.4 is essentially a calculation on the annulus $A$ and uses little about the enclosing surface $X$ except that it is foliated by closed trajectories of $\phi$. Indeed, the same argument can be applied with the compact surface $X$ replaced 
by an annulus $B$ and with $\phi=d z_{B}^{2}$ the natural quadratic differential on $B$, and it is this version we will need in the proof of Theorem 6.6.

Lemma 6.5 Let $B$ be an annular Riemann surface of finite modulus and $A \subset B$ a homotopically essential subannulus. Then

$$
E(B) \geq\left\langle\frac{d \overline{z_{A}}}{d z_{A}}, d z_{B}^{2}\right\rangle \geq \frac{2 E(B)^{2}}{E(A)}-E(B) .
$$

\section{Extremal length and grafting rays}

Using Lemma 6.4 as the main technical tool, we are now ready to prove Theorem 6.2.

Proof of Theorem 6.2 First, we consider the case when $\lambda=\gamma$ is a simple closed geodesic with unit weight. For brevity let $Y_{t}=\operatorname{gr}_{t \gamma} X$; we abbreviate $E(t)=E\left(\gamma, Y_{t}\right)$ and $\ell=\ell(\gamma, X)$.

The proof of (1) follows the usual pattern for an extremal length estimate (see Kerckhoff [22, Section 3]): a particular annulus homotopic to $\gamma$ bounds $E(t)$ from above, while a particular conformal metric on the surface bounds $E(t)$ from below. In this case the annulus is the grafting cylinder $A_{t} \subset Y_{t}$ of modulus $t / \ell$, and the conformal metric on $Y_{t}$ is the Thurston metric-the union of the product metric on $A_{t}=[0, t] \times \gamma$ and the hyperbolic metric of $X$ (see Tanigawa [42, Section 2.1]). Applying the geometric and analytic definitions of extremal length gives

$$
\frac{\ell}{t}>E\left(\gamma, Y_{t}\right)>\frac{\ell^{2}}{t \ell+A}>\frac{\ell}{t}-\frac{A}{t^{2}}
$$

where $A=4 \pi(g-1)$ and $(t \ell+A)$ is the area of the Thurston metric; thus (1) follows.

For statement (2), we must estimate the derivative of extremal length. For all $s, t>0$, there is a natural quasiconformal map from $Y_{t}$ to $Y_{s}$ that is affine on $A_{t}$, stretching it vertically in the natural coordinate, and conformal on $\left(Y_{t}-A_{t}\right)$. The derivative of this family of maps at $s=t$ is the Beltrami differential

$$
\mu(t)=-(2 t)^{-1} \frac{d \overline{z_{t}}}{d z_{t}}
$$

where $z_{t}$ is the natural coordinate on the grafting annulus $A_{t}$ and the Beltrami differential $d \overline{z_{t}} / d z_{t}$ is understood to be identically zero outside $A_{t}$.

By Theorem 6.3, the derivative of extremal length along the grafting ray is

$$
\frac{d}{d t} E(t)=2\langle\mu(t), \phi(t)\rangle=-t^{-1}\left\langle\frac{d \overline{z_{t}}}{d z_{t}}, \phi(t)\right\rangle .
$$


We estimate the pairing $\left\langle d \overline{z_{t}} / d z_{t}, \phi(t)\right\rangle$ using Lemma 6.4; starting with the upper bound, we have

$$
\left\langle\frac{d \overline{z_{t}}}{d z_{t}}, \phi(t)\right\rangle \leq E(t) \leq \frac{\ell}{t}
$$

while the lower bound from the lemma gives

$$
\left\langle\frac{d \overline{z_{t}}}{d z_{t}}, \phi(t)\right\rangle \geq \frac{2 E(t)^{2}}{E\left(A_{t}\right)}-E(t)=\frac{2 t}{\ell} E(t)^{2}-E(t) .
$$

Using $E(t) \geq \ell / t-A / t^{2}$ on the first term and $E(t) \leq \ell / t$ on the second, we obtain

$$
\left\langle\frac{d \overline{z_{t}}}{d z_{t}}, \phi(t)\right\rangle \geq \frac{2 t}{\ell}\left(\frac{\ell}{t}-\frac{A}{t^{2}}\right)^{2}-\frac{\ell}{t} \geq \frac{\ell}{t}-\frac{4 A}{t^{2}} .
$$

Multiplying (6-5) and (6-6) by $-1 / t$ and using the formula (6-4) for the derivative of extremal length, we have

$$
-\frac{\ell(\gamma, X)}{t^{2}} \leq \frac{d}{d t} E\left(\gamma, \mathrm{gr}_{t \gamma} X\right) \leq-\frac{\ell(\gamma, X)}{t^{2}}+\frac{4 A}{t^{3}}
$$

which gives part (2), completing the proof of Theorem 6.2 for simple closed geodesics.

The limiting arguments that extend (1) and (2) to general laminations are completely analogous, so we will only give details for the former. Given a measured lamination $\lambda \in \mathcal{M L}(S)$, define

$$
\delta(s, \lambda, X)=s^{2}\left|E\left(\lambda, \mathrm{gr}_{s \lambda} X\right)-\frac{\ell\left(\lambda, \mathrm{gr}_{s \lambda} X\right)}{s}\right|
$$

Since hyperbolic length, extremal length, and grafting are continuous on $\mathcal{M L}(S) \times$ $\mathcal{T}(S)$, this is a continuous nonnegative function $\delta: \mathbb{R}^{+} \times \mathcal{M L}(S) \times \mathcal{T}(S) \rightarrow \mathbb{R}$. Part (1) of the Theorem is equivalent to the statement that $\delta$ is bounded. We have shown that this is true for simple closed geodesics, ie there exists $C>0$ depending only on $\chi(S)$ such that for all simple closed curves $\gamma$ and all $s>0$, we have

$$
\delta(s, \gamma, X) \leq C
$$

Suppose that $\lambda=c \gamma$ is a weighted simple closed geodesic. Since hyperbolic length scales linearly in the transverse measure, while extremal length scales quadratically, 
we have

$$
\begin{aligned}
\delta(s, c \gamma, X) & =s^{2}\left|E\left(c \gamma, \mathrm{gr}_{s c \gamma} X\right)-\frac{\ell\left(c \gamma, \mathrm{gr}_{s c \gamma} X\right)}{s}\right| \\
& =s^{2} c^{2}\left|E\left(\gamma, \mathrm{gr}_{s c \gamma} X\right)-\frac{\ell\left(\gamma, \mathrm{gr}_{s c \gamma} X\right)}{s c}\right| \\
& =\delta(s c, \gamma, X) \leq C .
\end{aligned}
$$

Since weighted simple closed geodesics are dense in $\mathcal{M L}(S)$, and $\delta$ is continuous, this shows that $\delta \leq C$, establishing (1) for all measured laminations.

A similar limiting argument applies to part (2), where one bounds the error function

$$
\Delta(s, \lambda, X)=s^{3}\left|\left(\frac{d}{d s} E\left(\lambda, \operatorname{gr}_{s \lambda} X\right)\right)+\frac{\ell(\lambda, X)}{s^{2}}\right| .
$$

In this case, the function $\Delta$ in continuous because extremal length is $C^{1}$ on Teichmüller space (see Gardiner-Masur [17]), with its derivative varying continuously in $\mathcal{M L}(S)$, and since the derivatives of grafting rays depend continuously on the lamination (by Theorem 6.1).

As a consequence of Theorem 6.2, extremal length decreases along grafting rays outside of a compact set in $\mathcal{T}(S)$ of the form $\left\{\operatorname{gr}_{\lambda} X \mid \ell(\lambda, X) \leq L\right\}$.

\section{Hyperbolic length}

The hyperbolic length of $\lambda$ along a grafting ray is more difficult to control than the extremal length, but for the case of a single curve with large weight, the same techniques used in the proof of Theorem 6.2 give the following Theorem.

Theorem 6.6 Let $X \in \mathcal{T}(S)$ and let $\gamma$ be a simple closed hyperbolic geodesic on $X$. Then the hyperbolic length of $\gamma$ is of order $1 / t$ on the $\gamma$-grafting ray and is monotone decreasing for all $t \gg 0$. Specifically, we have

(1) $\ell\left(\gamma, \operatorname{gr}_{t \gamma} X\right)=\frac{\pi \ell(\gamma, X)}{t}+O\left(t^{-2}\right)$

(2) $\frac{d}{d t} \ell\left(\gamma, g_{t \gamma} X\right)=-\frac{\pi \ell(\gamma, X)}{t^{2}}+O\left(t^{-3}\right)$

as $t \rightarrow \infty$, where the implicit constants depend on $X$ and $\gamma$.

Note that Theorem 6.6 includes the results stated in the introduction as Theorem 1.5. 
Proof As before let $Y_{t}=\operatorname{gr}_{t \gamma} X$, and abbreviate $\ell(t)=\ell\left(\gamma, Y_{t}\right)$ and $E(t)=E\left(\gamma, Y_{t}\right)$.

A standard argument using the collar lemma shows that the extremal and hyperbolic length of a curve are asymptotically proportional when the length is small; specifically, we have

$$
\pi E(t)-C E(t)^{2}<\ell(t)<\pi E(t)
$$

for all $t$ such that $E(t)<1$, where $C$ is a universal constant. Since $E(t)<1$ for all $t>\ell(\gamma, X)$, substituting the estimate for $E(t)$ from Theorem 6.2 gives (1).

Now we establish the derivative estimate (2). Let $\hat{Y}_{t}$ denote the cover of $Y_{t}$ corresponding to the subgroup $\langle\gamma\rangle \subset \pi_{1}\left(Y_{t}\right)$. Since $\hat{Y}_{t}$ is conformally equivalent to an annulus of modulus $\pi / \ell(t)$, we have $\ell^{\prime}(t)=\pi \frac{d}{d t} E\left(\hat{Y}_{t}\right)$.

Recall that $\mu(t)=(-2 t)^{-1} d \overline{z_{t}} / d z_{t}$ represents the derivative of $Y_{t}$, where $z_{t}$ is the natural coordinate on $A_{t}$. Thus the derivative of the annular covers $\hat{Y}_{t}$ is represented by the pullback Beltrami differential $p^{*}(\mu(t))$ where $p: \hat{Y}_{t} \rightarrow Y_{t}$ is the covering projection. Let $w_{t}$ denote the natural coordinate on $\hat{Y}_{t}$. By (6-1) we have

$$
\ell^{\prime}(t)=\pi \frac{d}{d t} E\left(\hat{Y}_{t}\right)=2 \pi\left\langle p^{*}(\mu(t)), d w_{t}^{2}\right\rangle .
$$

To estimate this pairing, we analyze the differential $p^{*}(\mu(t))$; its support is the preimage of the grafting cylinder $p^{-1}\left(A_{t}\right) \subset \hat{Y}_{t}$, which consists of

(i) a homotopically essentially annulus $\hat{A}_{t}$ such that $\left.p\right|_{\widehat{A}_{t}}: \widehat{A}_{t} \rightarrow A_{t}$ is a conformal isomorphism, and

(ii) a complementary set $\Omega=p^{-1}\left(A_{t}\right)-\hat{A}_{t}$ that is a disjoint union of countably many simply connected regions $\Sigma_{i} \subset \hat{Y}_{t}$ such that the restriction of $p$ to any one of them gives a universal covering $\left.p\right|_{\Sigma_{i}}: \Sigma_{i} \rightarrow A_{t}$,

as depicted in Figure 4. Therefore we have

$$
\left\langle p^{*}(\mu(t)), d w_{t}^{2}\right\rangle=\int_{\widehat{A}_{t}} p^{*}(\mu(t)) d w_{t}^{2}+\int_{\Omega} p^{*}(\mu(t)) d w_{t}^{2},
$$

and we can analyze these two terms individually.

By the length estimates of part (1), the reciprocal moduli $E\left(\hat{A}_{t}\right)=E\left(A_{t}\right)=\ell(0) / t$ and $E\left(\hat{Y}_{t}\right)=\ell(t) / \pi$ differ by $O\left(t^{-2}\right)$. It follows that the subannulus $\hat{A}_{t}$ accounts for nearly all of the area of $\hat{Y}_{t}$. In fact, restricting the metric $\left|d w_{t}\right|$ to $\hat{A}_{t}$ and using the analytic definition of extremal length gives

$$
\operatorname{Area}\left(\hat{A}_{t},\left|d w_{t}\right|\right) \geq \frac{\ell\left(\gamma,\left|d w_{t}\right|\right)^{2}}{E\left(\hat{A}_{t}\right)}=\frac{E\left(\hat{Y}_{t}\right)^{2}}{E\left(\hat{A}_{t}\right)} \geq \frac{\ell(0)}{t}-\frac{C}{t^{2}},
$$




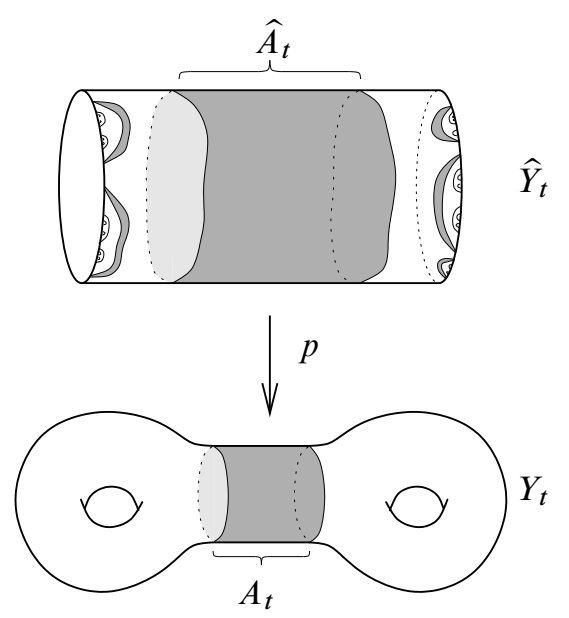

Figure 4: The grafting annulus and its lifts to the annular cover.

where $C$ depends on $\gamma$ and $X$. Since $\operatorname{Area}\left(\widehat{Y}_{t},\left|d w_{t}\right|\right)=\ell(t) / \pi<\ell(0) / t$ and $\Omega$ is disjoint from $\hat{A}_{t}$, we have $\operatorname{Area}\left(\Omega,\left|d w_{t}\right|\right) \leq C / t^{2}$.

This area estimate implies that the second term in (6-9) is negligible, ie

$$
\begin{aligned}
\left|\int_{\Omega} p^{*}(\mu(t)) d w_{t}^{2}\right| & \leq\left\|p^{*}(\mu(t))\right\|_{\infty} \operatorname{Area}\left(\Omega,\left|d w_{t}\right|\right) \\
& \leq\left(\frac{1}{2 t}\right)\left(\frac{C_{2}}{t^{2}}\right)=O\left(t^{-3}\right)
\end{aligned}
$$

Now we consider the first term in (6-9). Since $\left.p\right|_{\hat{A}_{t}}$ is a conformal isomorphism, we have $\left.p^{*}(\mu(t))\right|_{\hat{A}_{t}}=(-2 t)^{-1} d \overline{\hat{z}}_{t} / d \hat{z}_{t}$ where $\hat{z}_{t}$ is the natural coordinate of $\widehat{A}_{t}$. Applying Lemma 6.5 we have

$$
E\left(\widehat{Y}_{t}\right) \geq\left\langle\frac{d \bar{z}_{t}}{d \widehat{z}_{t}}, d w_{t}^{2}\right\rangle \geq \frac{2 E\left(\hat{Y}_{t}\right)^{2}}{E\left(\hat{A}_{t}\right)}-E\left(\hat{Y}_{t}\right)
$$

As before we substitute $E\left(\hat{Y}_{t}\right)=\ell(t) / \pi, E\left(\hat{A}_{t}\right)=\ell(0) / t$, and apply the estimates for $\ell(t)$ to obtain

$$
\int_{\widehat{A}_{t}} p^{*}(\mu(t)) d w_{t}^{2}=(-2 t)^{-1}\left\langle\frac{d \widehat{z}_{t}}{d \widehat{z}_{t}}, d w_{t}^{2}\right\rangle=-\frac{\ell(0)}{2 t^{2}}+O\left(t^{-3}\right) .
$$


Thus we have estimates for both terms in (6-9), and applying the formula (6-8) for $\ell^{\prime}(t)$ gives the desired result:

$$
\ell^{\prime}(t)=-\frac{\pi \ell(0)}{t^{2}}+O\left(t^{-3}\right)
$$

Remark In [29, Corollary 3.2], McMullen shows that $\ell\left(\lambda, \operatorname{gr}_{t \lambda} X\right)<\ell(\lambda, X)$ for all $X \in \mathcal{T}(S), \lambda \in \mathcal{M L}(S)$ and $t>0$; it is also mentioned that this upper bound can be strengthened to

$$
\ell\left(\gamma, \operatorname{gr}_{t \gamma} X\right) \leq \frac{\pi}{\pi+t} \ell(\gamma, X)
$$

for any simple closed curve $\gamma$. For details on this and a corresponding lower bound, see [10, Proposition 3.4]. Theorem 6.6 shows that this upper bound is asymptotically sharp.

\section{References}

[1] L Bers, Simultaneous uniformization, Bull. Amer. Math. Soc. 66 (1960) 94-97 MR0111834

[2] L Bers, Correction to "Spaces of Riemann surfaces as bounded domains", Bull. Amer. Math. Soc. 67 (1961) 465-466 MR0130972

[3] F Bonahon, Earthquakes on Riemann surfaces and on measured geodesic laminations, Trans. Amer. Math. Soc. 330 (1992) 69-95 MR1049611

[4] F Bonahon, Shearing hyperbolic surfaces, bending pleated surfaces and Thurston's symplectic form, Ann. Fac. Sci. Toulouse Math. (6) 5 (1996) 233-297 MR1413855

[5] F Bonahon, Geodesic laminations with transverse Hölder distributions, Ann. Sci. École Norm. Sup. (4) 30 (1997) 205-240 MR1432054

[6] F Bonahon, Transverse Hölder distributions for geodesic laminations, Topology 36 (1997) 103-122 MR1410466

[7] F Bonahon, Variations of the boundary geometry of 3-dimensional hyperbolic convex cores, J. Differential Geom. 50 (1998) 1-24 MR1678469

[8] F Bonahon, Kleinian groups which are almost Fuchsian, J. Reine Angew. Math. 587 (2005) 1-15 MR2186972

[9] F Bonahon, J-P Otal, Laminations measurées de plissage des variétés hyperboliques de dimension 3, Ann. of Math. (2) 160 (2004) 1013-1055 MR2144972

[10] R Díaz, I Kim, Asymptotic behavior of grafting rays (2007) Preprint

[11] D Dumas, Grafting, pruning, and the antipodal map on measured laminations, J. Differential Geom. 74 (2006) 93-118 MR2260929 Erratum, J. Differential Geom. 77 (2007) 175-176 
[12] D Dumas, The Schwarzian derivative and measured laminations on Riemann surfaces, Duke Math. J. 140 (2007) 203-243 MR2359819

[13] D B A Epstein, A Marden, Convex hulls in hyperbolic space, a theorem of Sullivan, and measured pleated surfaces, from: "Analytical and geometric aspects of hyperbolic space (Coventry/Durham, 1984)", London Math. Soc. Lecture Note Ser. 111, Cambridge Univ. Press, Cambridge (1987) 113-253 MR903852

[14] D B A Epstein, A Marden, V Markovic, Quasiconformal homeomorphisms and the convex hull boundary, Ann. of Math. (2) 159 (2004) 305-336 MR2052356

[15] D Gallo, M Kapovich, A Marden, The monodromy groups of Schwarzian equations on closed Riemann surfaces, Ann. of Math. (2) 151 (2000) 625-704 MR1765706

[16] F P Gardiner, Teichmüller theory and quadratic differentials, Pure and Applied Mathematics (New York), John Wiley \& Sons, New York (1987) MR903027 A WileyInterscience Publication

[17] F P Gardiner, H Masur, Extremal length geometry of Teichmüller space, Complex Variables Theory Appl. 16 (1991) 209-237 MR1099913

[18] W M Goldman, Projective structures with Fuchsian holonomy, J. Differential Geom. 25 (1987) 297-326 MR882826

[19] D A Hejhal, Monodromy groups and linearly polymorphic functions, Acta Math. 135 (1975) 1-55 MR0463429

[20] Y Kamishima, S P Tan, Deformation spaces on geometric structures, from: "Aspects of low-dimensional manifolds", Adv. Stud. Pure Math. 20, Kinokuniya, Tokyo (1992) 263-299 MR1208313

[21] A B Katok, Invariant measures of flows on orientable surfaces, Dokl. Akad. Nauk SSSR 211 (1973) 775-778 MR0331438

[22] S P Kerckhoff, The asymptotic geometry of Teichmüller space, Topology 19 (1980) 23-41 MR559474

[23] S P Kerckhoff, Earthquakes are analytic, Comment. Math. Helv. 60 (1985) 17-30 MR787659

[24] S P Kerckhoff, Simplicial systems for interval exchange maps and measured foliations, Ergodic Theory Dynam. Systems 5 (1985) 257-271 MR796753

[25] F Klein, Vorlesungen über die Hypergeometrische funktion, Springer-Verlag, Berlin (1933)

[26] G Levitt, Feuilletages des surfaces, Ann. Inst. Fourier (Grenoble) 32 (1982) x, 179217 MR662443

[27] B Maskit, On a class of Kleinian groups, Ann. Acad. Sci. Fenn. Ser. A I No. 442 (1969) 8 MR0252638 
[28] H Masur, Interval exchange transformations and measured foliations, Ann. of Math. (2) 115 (1982) 169-200 MR644018

[29] C T McMullen, Complex earthquakes and Teichmüller theory, J. Amer. Math. Soc. 11 (1998) 283-320 MR1478844

[30] S Nag, The complex analytic theory of Teichmüller spaces, Canadian Mathematical Society Series of Monographs and Advanced Texts, John Wiley \& Sons, New York (1988) MR927291 A Wiley-Interscience Publication.

[31] J-P Otal, Le théorème d'hyperbolisation pour les variétés fibrées de dimension 3, Astérisque (1996) x+159 MR1402300

[32] A Papadopoulos, Deux remarques sur la géométrie symplectique de l'espace des feuilletages mesurés sur une surface, Ann. Inst. Fourier (Grenoble) 36 (1986) 127-141 MR850748

[33] R C Penner, J L Harer, Combinatorics of train tracks, Annals of Mathematics Studies 125, Princeton University Press, Princeton, NJ (1992) MR1144770

[34] I D Platis, Complex symplectic geometry of quasi-Fuchsian space, Geom. Dedicata 87 (2001) 17-34 MR1866841

[35] M Rees, An alternative approach to the ergodic theory of measured foliations on surfaces, Ergodic Theory Dynamical Systems 1 (1981) 461-488 (1982) MR662738

[36] K P Scannell, M Wolf, The grafting map of Teichmüller space, J. Amer. Math. Soc. 15 (2002) 893-927 MR1915822

[37] C Series, On Kerckhoff minima and pleating loci for quasi-Fuchsian groups, Geom. Dedicata 88 (2001) 211-237 MR1877217

[38] C Series, Limits of quasi-Fuchsian groups with small bending, Duke Math. J. 128 (2005) 285-329 MR2140265

[39] C Series, Thurston's bending measure conjecture for once punctured torus groups, from: "Spaces of Kleinian groups", London Math. Soc. Lecture Note Ser. 329, Cambridge Univ. Press, Cambridge (2006) 75-89 MR2258745

[40] Y Sözen, F Bonahon, The Weil-Petersson and Thurston symplectic forms, Duke Math. J. 108 (2001) 581-597 MR1838662

[41] D Sullivan, W Thurston, Manifolds with canonical coordinate charts: some examples, Enseign. Math. (2) 29 (1983) 15-25 MR702731

[42] H Tanigawa, Grafting, harmonic maps and projective structures on surfaces, J. Differential Geom. 47 (1997) 399-419 MR1617652

[43] WP Thurston, Minimal stretch maps between hyperbolic surfaces arXiv: math.GT/9801039

[44] W P Thurston, Geometry and Topology of Three-Manifolds, Princeton lecture notes (1979) Available at http://www.msri.org/publications/books/gt3m/ 
[45] W P Thurston, Earthquakes in two-dimensional hyperbolic geometry, from: "Lowdimensional topology and Kleinian groups (Coventry/Durham, 1984)", London Math. Soc. Lecture Note Ser. 112, Cambridge Univ. Press, Cambridge (1986) 91-112 MR903860

[46] A J Tromba, Teichmüller theory in Riemannian geometry, Lectures in Mathematics ETH Zürich, Birkhäuser Verlag, Basel (1992) MR1164870 Lecture notes prepared by Jochen Denzler.

[47] W A Veech, Gauss measures for transformations on the space of interval exchange maps, Ann. of Math. (2) 115 (1982) 201-242 MR644019

[48] S Wolpert, An elementary formula for the Fenchel-Nielsen twist, Comment. Math. Helv. 56 (1981) 132-135 MR615620

[49] S Wolpert, The Fenchel-Nielsen deformation, Ann. of Math. (2) 115 (1982) 501-528 MR657237

[50] S Wolpert, On the symplectic geometry of deformations of a hyperbolic surface, Ann. of Math. (2) 117 (1983) 207-234 MR690844

[51] S Wolpert, Chern forms and the Riemann tensor for the moduli space of curves, Invent. Math. 85 (1986) 119-145 MR842050

[52] P G Zograf, L A Takhtadzhyan, On the Liouville equation, accessory parameters and the geometry of Teichmüller space for Riemann surfaces of genus 0, Mat. Sb. (N.S.) 132(174) (1987) 147-166 MR882831

Department of Mathematics, Brown University

Providence RI 02912, USA

Department of Mathematics, Rice University

Houston TX 77005, USA

ddumas@math.brown.edu, mwolf@math.rice.edu

http://www.math.brown.edu/ ddumas/, http://www.math.rice.edu/ mwolf/

Proposed: Jean-Pierre Otal

Received: 23 April 2007

Seconded: Benson Farb, Martin Bridson

Accepted: 20 August 2007

Geometry 8 Topology, Volume 12 (2008) 